\title{
Repurposed Drugs Block Toxin-Driven Platelet Clearance by the Hepatic Ashwell- Morell Receptor to Clear Staphylococcus aureus Bacteremia
}

\author{
Josh Sun ${ }^{1,2,3^{*}}$, Satoshi Uchiyama ${ }^{1 *}$, Joshua Olson ${ }^{1}$, Yosuke Morodomi $^{4}$, Ingrid Cornax ${ }^{1}$, Nao Ando ${ }^{1}$, \\ Yohei Kohno ${ }^{1}$, May M. T. Kyaw ${ }^{1}$, Bernice Aguilar ${ }^{1}$, Nina M. Haste ${ }^{1,2,3}$, Sachiko Kanaji ${ }^{4}$, Taisuke \\ Kanaji ${ }^{4}$, Warren E. Rose ${ }^{5}$, George Sakoulas ${ }^{2}$, Jamey D. Marth ${ }^{6,7}$, Victor Nizet ${ }^{1,2,3 \dagger}$
}

${ }^{1}$ Biomedical Sciences Graduate Program, UC San Diego, La Jolla, CA 92093, USA

${ }^{2}$ Department of Pediatrics, UC San Diego, La Jolla, CA 92093, USA

${ }^{3}$ Skaggs School of Pharmacy and Pharmaceutical Sciences, UC San Diego, La Jolla, CA 92093, USA

${ }^{4}$ Department of Molecular Medicine, MERU-Roon Research Center on Vascular Biology, The Scripps Research Institute, La Jolla, CA, USA

${ }^{5}$ School of Pharmacy, University of Wisconsin, Madison, WI 53705, USA

${ }^{6}$ Center for Nanomedicine, UC Santa Barbara, Santa Barbara, CA 93106, USA

${ }^{7}$ Sanford Burnham Prebys Medical Discovery Institute, UC Santa Barbara, Santa Barbara, CA 93106 USA

${ }^{*}$ These authors contributed equally

†Correspondence: Victor Nizet (vnizet@ucsd.edu) 


\begin{abstract}
Staphylococcus aureus (SA) bloodstream infections cause high morbidity and mortality (20-30\%) despite modern supportive care. In a human bacteremia cohort, development of thrombocytopenia was correlated to increased mortality and increased $\alpha$-toxin expression by the pathogen. Platelet-derived antibacterial peptides are important in bloodstream defense against SA, but $\alpha$-toxin decreased platelet viability, induced platelet sialidase to cause desialylation of platelet glycoproteins, and accelerated platelet clearance by the hepatic Ashwell-Morell receptor (AMR). Ticagrelor $\left(\right.$ Brilinta $\left.^{\circledR}\right)$, a commonly prescribed P2Y12 receptor inhibitor used post-myocardial infarction, blocked $\alpha$-toxin-mediated platelet injury and resulting thrombocytopenia, thus providing protection from lethal SA infection in a murine intravenous challenge model. Genetic deletion or pharmacological inhibition of AMR stabilized platelet counts and enhanced resistance to SA infection, and the anti-influenza sialidase inhibitor oseltamivir $\left(\right.$ Tamiflu $\left.^{\circledR}\right)$ provided similar therapeutic benefit. Thus a "toxin-platelet-AMR" regulatory pathway plays a critical role in the pathogenesis of SA bloodstream infection, and its elucidation provides proof-of-concept for repurposing two FDA-approved drugs as adjunctive therapies to improve patient outcomes.
\end{abstract}




\section{INTRODUCTION}

Staphylococcus aureus (SA) is one of the most important human bacterial pathogens, as the second leading cause of bloodstream infections (bacteremia) and the leading cause of infective endocarditis (1). Despite modern supportive measures, overall mortality in SA bacteremia has not declined in decades and remains unacceptably high (20-30\%), with significant risk of complications including sepsis syndrome, endocarditis and metastatic foci of infection (e.g. osteomyelitis) (2). High risk populations include the elderly, diabetics, surgical and hemodialysis patients (3). Multidrug resistance (e.g. methicillin-resistant SA, MRSA) is prevalent and associated with adverse outcome and increased medical costs (4).

The high incidence of SA bacteremia signifies a remarkable capacity of the organism to resist host innate defense mechanisms that function to prevent pathogen bloodstream dissemination (5). Extensive research has focused on SA virulence factors that counteract opsonization by serum complement (6), surfaceanchored protein A that impairs Fc function of antibodies (7), and the pathogen's numerous resistance mechanisms to avoid phagocytosis and oxidative burst killing by neutrophils (8).

Comparatively less is understood about how SA interacts with circulating platelets. These abundant, small anucleate cells are best known for their central role in hemostasis, but increasingly appreciated to possess bioactivities relevant to immune defense (9). Platelets can act as mechano-scavengers to bundle bacteria (10) and enhance the function of professional phagocytic cell types such as neutrophils (11), macrophages (12) and hepatic Kuppfer cells $(13,14)$. Platelets express several Toll-like receptors that recognize pathogen-associated molecular patterns (15) to activate their release of pro-inflammatory cytokines (e.g. interleukin-1ß) (16) and antimicrobial peptides including platelet microbicidal protein (tPMP) and human beta-defensin-1 (hBD-1) with direct antibacterial actions $(12,17,18)$. SA activates platelets via integrin GP11b/IIIa, Fc $\gamma$ RIIa receptor and ADAM-10-dependent pathways (19-21), and the pathogen induces platelet aggregation via its "clumping factors" $\mathrm{ClfA}$ and $\mathrm{ClfB}(22,23)$. 
In a cohort of 49 patients with SA bacteremia, we report a strong association of mortality with lowered platelet count (thrombocytopenia) rather than changes in leukocyte count. Of note, platelet depletion in mice was recently shown to impair SA clearance $(12,24)$. Our mechanistic analysis with human platelets ex vivo and murine platelets in vivo revealed a critical activity of platelets in direct killing of SA. The pathogen attempts to counteract this defense by deploying a pore-forming toxin ( $\alpha$-toxin) to (i) disrupt platelet antimicrobial activity and (ii) accelerate sialidase-dependent platelet clearance through the hepatic Ashwell-Morell receptor (AMR). Elucidation of this "toxin-platelet-AMR" regulatory pathway guided us to therapeutic repurposing of two FDA-approved drugs to preserve platelet homeostasis, thereby providing significant host protection in experimental SA bloodstream infection.

\section{RESULTS}

\section{Platelets are essential for blood immunity against SA bacteremia}

The normal human platelet count ranges from $150,000-450,000 / \mathrm{mm}^{3}$ of blood. In 49 consecutive patients with SA bacteremia (blood culture growing MRSA or MSSA) identified prospectively at an academic medical center in Madison, WI (25), we observed a strong association of patient mortality with thrombocytopenia (platelet count $<100,000 / \mathrm{mm}^{3}$ ) on the initial blood sample and not abnormally elevated or reduced leukocyte count (Fig. 1, A and B). Indeed, two patients with thrombocytopenia failed to clear their bacteremia despite antibiotic therapy for more than 60 days before succumbing. No significant correlation was observed between platelet count and serum levels of pro-inflammatory cytokine IL-1 $\beta$ nor APACHE score, a clinical metric of disease severity (Fig. 1A). These data are consistent with a prior single center study in Israel showing thrombocytopenia (and not leukocyte count) to be a significant risk factor for 30-day all-cause mortality in SA bacteremia, although overall mortality rates were much higher than other published series (56.2\% in thrombocytopenic group vs. $34 \%$ with normal platelets counts) (26). These 
clinical studies suggest that circulating platelets, and not white blood cells, could play the dominant role in resolution of SA during bloodstream infection. Indeed, when directly compared, the second most common Gram-positive bacterial pathogen associated with human bacteremia, Streptococcus pneumoniae (1), SA was $\sim 50 \%$ more resistant to killing by purified human neutrophils, but $\sim 70 \%$ more susceptible to killing by human platelets (Fig. 1C).

We pursued this association further using an in vivo model of SA bacteremia (MRSA strain USA300 TCH1516) established by intravenous (i.v.) tail vein injection in mice, where normal platelet count ranges from 900,000 to 1.4 million cells $/ \mathrm{mm}^{3}$ blood (27). Within $2 \mathrm{~h}$ of SA challenge, the circulating platelet count of infected mice was reduced by $\sim 40 \%$ from baseline levels $(1237 \pm 49.82$ vs. $772 \pm 49.91)$ (Fig. 1D). To verify that reduced platelet count was indicative of a functional immune deficiency, we used an anti-CD41 antibody to deplete mice of platelets to $17 \%$ of baseline levels (fig. S1). The drawn blood of the thrombocytopenic animals was impaired in ex vivo killing of SA (67.5 $\pm 7.9 \%$ surviving CFU vs. $25 \pm 2.1 \%$ surviving CFU in normal blood) (Fig. 1E), and bacterial burdens in blood and kidneys of thrombocytopenic mice were significantly increased vs. untreated mice within 2 h following i.v. SA challenge (Fig. 1F).

\section{SA $\alpha$-toxin induces thrombocytopenia to evade platelet-mediated microbicidal activity}

A major SA secreted virulence factor, the pore-forming $\alpha$-toxin (Hla), induces platelet cytotoxicity and aberrant aggregation after binding its protein receptor A-disintegrin metalloprotease-10 (ADAM-10) on the platelet surface $(14,20,28)$. Using ImageJ densitometric analysis of anti-Hla western immunoblots, we grouped the SA bacteremia isolates from our clinical cohort into low- $(n=18)$, medium- $(n=15)$, and high$(\mathrm{n}=16) \alpha$-toxin producers (fig. S2). A significant correlation was seen between high-level $\alpha$-toxin production and thrombocytopenia (Fig. 1G), compared to the low-level and medium-level $\alpha$-toxin producing groups. There was no significant correlation between $\alpha$-toxin production and leukocyte counts (Fig. 1H) For comparison to the wild-type (WT) parent SA strain in analyses of platelet interactions, we constructed a 
$\Delta h l a$ knockout strain by precise allelic replacement (fig. S3). In the mouse i.v. challenge model, the SA Shla mutant induced less thrombocytopenia (Fig. 1I) and was more rapidly cleared from the blood circulation (Fig. 1J) than the WT parent strain. Ex vivo, the SA $\Delta$ hla mutant was more susceptible to killing by purified human platelets (Fig. 1K). Together these studies indicate that the virulence effects of SA $\alpha$ toxin extend to evasion of direct platelet-mediated antibacterial killing.

\section{FDA-approved P2Y12 inhibitor ticagrelor blocks SA $\alpha$-toxin-mediated platelet cytotoxicity}

Inhibition of platelet activation is the target of antithrombotic drug therapy designed to reduce the risk of cardiovascular death, myocardial infarction (MI), and stroke in patients with acute coronary syndrome or a history of MI, beginning with classical studies of aspirin (acetylsalicylic acid, ASA) in the 1970s, then extending to newer selective inhibitors of adenosine signaling through the platelet P2Y12 receptor (clopidogrel, prasugrel, ticagrelor) (29). However, the effect of "antiplatelet" drugs on the direct antibacterial properties of platelets has not been reported. Of potential relevance, a clinical study of 224 consecutive patients with community-acquired pneumonia found that those receiving antiplatelet therapy (ASA and/or clopidogrel) for secondary prevention of cardiovascular disease had reduced need for intensive care unit treatment (odds ratio $0.19,95 \%$ confidence interval $0.04-0.87)$ and shorter hospital stays $(13.9 \pm$ 6.2 vs. $18.2 \pm 10.2$ days) compared to their age-matched cohort (30). Additional human retrospective or matched cohort studies of endocarditis, bacteremia or sepsis (not restricted to SA) have provided similar hints of improved clinical outcome among patients receiving antiplatelet drugs (31-34).

To discriminate the effect of the two antiplatelet drug classes on SA killing, we pretreated freshly isolated human platelets for 15 min with ASA or ticagrelor (chosen since clopidogrel is a prodrug requiring hepatic conversion in vivo) and co-incubated them with the bacteria. Within $2 \mathrm{~h}$, ticagrelor-treated platelets showed a 2.2-fold enhancement in SA killing vs. untreated controls (Fig. 2A), whereas ASA did not significantly alter platelet antibacterial activity. In contrast, ticagrelor did not promote macrophage or neutrophil killing 
of SA, did not alter neutrophil extracellular trap production, and did not directly inhibit SA growth (fig. S4, A-D). Upon direct co-incubation of SA with human platelets in a tissue culture well, severe platelet damage was evident by transmission electron microscopy; however, ticagrelor treatment preserved platelet structural integrity against SA-induced injury (Fig. 2B). As $\alpha$-toxin is the principal driver of SA platelet toxicity, we measured $\alpha$-toxin-induced lactate dehydrogenase (LDH) release from platelets treated with ticagrelor, ASA (COX-1 inhibitor), or specific small molecule inhibitors of other known platelet activation receptors (CD41, PAR-1, PAR4). Among these agents, only ticagrelor significantly inhibited SA $\alpha$-toxininduced platelet LDH release (Fig. 2C), doing so in a dose-dependent manner (Fig. 2D). The deleterious effect of $\alpha$-toxin on platelets involves activation of its receptor protease ADAM-10 leading to intracellular $\mathrm{Ca} 2+$ mobilization (20), and these biological effects were both significantly inhibited by ticagrelor as measured in specific assays (Fig. 2, $\mathbf{E}$ and F). While ticagrelor did not alter the amount of ADAM-10 expressed on the platelet surface (Fig. 2G), the P2Y12 inhibitor drug blocked SA-induced ADAM-10dependent shedding of platelet glycoprotein-6 (GP6) (Fig. 2H). SA exposure also induced P-selectin, a transmembrane protein specific to alpha granules that is translocated to the platelet surface upon activation (Fig. 2I), and raised surface levels of CD63, a marker of dense granule mobilization (fig. S5A). The SAinduced upregulation of P-selectin and CD63 were both blocked upon ticagrelor treatment (Fig. 2I, fig. S5A). Neither SA nor ticagrelor significantly affected platelet beta-galactosidase activity, another lysosomal marker (fig. S5B).

\section{FDA-approved P2Y12 inhibitor ticagrelor protects against SA bacteremia}

Inhibition of $\alpha$-toxin mediated platelet cytotoxicity suggested that P2Y12 inhibition using ticagrelor could mitigate the toxin's virulence role in driving SA-induced thrombocytopenia (Fig. 1I) to promote bloodstream survival of the pathogen (Fig. 1J). IV SA challenge in mice drove down platelet counts beginning as early as $4 \mathrm{~h}$ (35\% decrease) and continuing through $24 \mathrm{~h}$ (63\% decrease), with partial recovery by $72 \mathrm{~h}$ (35\% decrease) (fig. S6A); bone marrow analysis at $72 \mathrm{~h}$ revealed increased thrombopoiesis as 
evidenced by greater megakaryocyte number and by ploidy distribution (fig. S6B and C). The rapid SAinduced thrombocytopenia was associated with platelet GP6 shedding (fig. S6D and E) and platelet microparticulation (fig. S6F), the latter determined by in vitro studies to be $\alpha$-toxin-dependent (fig. S6G). Indeed, mice treated with ticagrelor maintained higher numbers of circulating platelets compared to control animals following SA i.v. infection (Fig. 3A), significantly reducing the bacterial burden in the blood (Fig. 3B) in systemic organs (kidney, liver, spleen, Fig, 3C), and ultimately improving survival in a 10-day mortality study (Fig. 3D). Blinded histological examination of tissues by a veterinary pathologist revealed the most striking treatment-associated differences in the kidneys (Fig. 3E) and the heart (fig. S7). A 4- to 10-fold reduction in bacterial micro-abscesses was identified within the renal glomeruli, tubules, and blood vessels of ticagrelor-treated mice vs. PBS control animals, corroborating the CFU quantification data. Renal microabscesses in the control group were generally larger and more densely packed with bacteria, while those in ticagrelor-treated mice were frequently disrupted by immune cell infiltrates. Together these data suggest that platelet P2Y12 inhibition blocks $\alpha$-toxin and SA-mediated platelet cytotoxicity and consequent thrombocytopenia, thus enhancing the platelet-mediated clearance of the pathogen in vitro and in vivo.

\section{SA $\alpha$-toxin activates endogenous platelet sialidase activity}

Our clinical data (Fig. 1A) and those of others (26), coupled with our experimental work (Figs. 1, E and F) and prior platelet depletion studies $(12,24)$, suggest platelet count per se is important in determining SA clinical outcome. Since $\alpha$-toxin production correlated to thrombocytopenia in patients (Fig. 1G) and in experimental mouse infection (Fig. 1I), we hypothesized that SA deploys the toxin as a means to deplete the host of an effective circulating innate immune cell. Yet, platelet senescence and clearance are tightly regulated by multiple mechanisms, in particular the highly conserved hepatic transmembrane heterodimeric Aswell-Morell receptor (AMR) (35). The AMR clears "aging” platelets with reduced terminal $\alpha 2,3-$ linked sialic acids on their surface glycoproteins and glycolipids by engaging the exposed underlying galactose. 
We asked if the observed therapeutic effect of ticagrelor in SA sepsis was solely based on inhibiting platelet cytotoxicity or may further intersect with this important mechanism of platelet homeostasis.

To assess platelet sialylation state during SA bacteremia, we obtained frozen plasma from 10 randomly selected adult patients with SA bacteremia, 10 patients with Escherichia coli bacteremia, and 5 healthy controls. We found there was a significant increase in exposed galactose (indicative of desiaylation) on the platelets of SA-infected patients compared to the two other groups (Fig. 4A). However, SA lacks a bacterial sialidase (neuraminidase) present in other pathogens including $S$. pneumoniae (Fig. 4B). Rather, we discovered that WT SA induces sialidase activity on purified human platelets, whereas its isogenic $\Delta$ hla mutant derivative did not (Fig. 4C). An increase in platelet sialidase activity in response to WT SA or purified $\alpha$-toxin, present within the platelet pellet but not released into the media, was detected in independent assays using lectin affinity and a fluorescent substrate (fig. S8A, Fig. 4D). Although the precise mechanism of its transfer is not established, Neul is the main endogenous sialidase that translocates from lysosomal stores to the platelet surface to target glycoproteins and expose AMR ligands (galactose) (36, 37), and we confirmed its upregulation in response to SA challenge by flow cytometry (fig. S8B). Probing the observed therapeutic effect of P2Y12 inhibition in this context, we found ticagrelor strongly inhibited SA-induced platelet sialidase activity (Fig. 4E). These results suggest that SA $\alpha$-toxin-induced thrombocytopenia may not depend on wholescale platelet injury, but instead involve accelerated hepatic AMR-dependent clearance of desialylated platelets upon surface mobilization of Neu1. Binding of ADP to P2Y12 elevates cytosolic calcium $\left(\mathrm{Ca}^{2+}\right)$ levels by stimulating phospholipase $\mathrm{C}$-mediated production of inositol-1,4,5-trisphosphate (IP3), which in turn releases $\mathrm{Ca}^{2+}$ from the intracellular stores through IP3 receptor channels. Because $\mathrm{Ca}^{2+}$ is a major signaling molecule that allows for ADP-induced lysosomal secretion, P2Y12 inhibition in theory would block this process. However, a singular correlation between $\mathrm{Ca}^{2+}$ levels and granular secretion remains ambiguous, as our data, as well as older literature, suggest that there are both $\mathrm{Ca}^{2+}$-dependent and $\mathrm{Ca}^{2+}$-independent granular secretory pathways $(38,39)$. That said, by 
linking sialidase activity to the ticagrelor therapeutic effect, additional target points for pharmacological support of platelet defense against SA came into view.

Inhibition of the hepatic Ashwell-Morell receptor (AMR) supports platelet-mediated defense against

\section{SA bacteremia}

In previous work, we showed that moderate thrombocytopenia mediated by AMR-dependent clearance of desialylated platelets was protective in experimental sepsis caused by $S$. pneumoniae, a sialidase-expressing pathogen (40, 41). However, as shown earlier (Fig. 1C), S. pneumoniae is resistant to human platelet killing, so removal of desialylated and hypercoagulable platelets does not deplete the bloodstream of an effective antimicrobial effector cell type. We asked whether the innate immune calculus could prove different for platelet-sensitive SA by challenging WT and AMR-deficient (Asgr2 $2^{-/}$) mice in the C57b1/6 background. Whereas WT mice remained highly sensitive to SA $\alpha$-toxin-induced thrombocytopenia, platelet counts in Asgr $2^{-/-}$mice did not drop following the bacterial challenge (Fig. 5A), indicating that recruitment of AMR clearance was the main pathogenic driver of platelet clearance during infection. And in striking contrast to the findings in S. pneumoniae infection (40), Asgr $2^{-/-}$mice, which are resistant to pathogen-induced thrombocytopenia, exhibited a strong survival advantage against lethal SA challenge (Fig. 5B). This genetic association could be reproduced pharmacologically in WT mice, where asialofetuin, a competitive glycoprotein inhibitor of the hepatic AMR (Fig. 5C), improved mouse survival in lethal SA challenge by maintaining platelet count during infection (Fig. 5D) and by reducing bacterial burden in the kidney, livers, and spleen (Fig. 5E). Corroborating that $\alpha$-toxin-dependent desialylation is driving the accelerated platelet clearance, no SA-induced reduction in platelet count was seen in mice lacking the hepatic AMR (Fig. 5A, Fig. 5F).

FDA-approved sialidase inhibitor oseltamivir blocks AMR-mediated platelet clearance and protects against SA bacteremia 
The above results showed that mice were protected against SA infection by ticagrelor, which inhibits $\alpha$ toxin-induced platelet desialylation, or by genetic or pharmacological inactivation of the AMR, which blocks hepatic clearance of desialylated platelets. Further corroboration of the importance of platelet sialylation for maintaining bloodstream defense against SA bacteremia was obtained using mice lacking the St3gal4 sialyltransferase gene, which show diminished platelet sialylation and baseline thrombocytopenia (42). Compared to WT C57bl6 mice, the St3gal44 mice had accelerated mortality upon IV SA infection (Fig. 6A), but no further SA-induced reduction in their already low platelet counts $(\sim 25 \%$ of normal mice, Fig. 6B). Since sialidase (Neu1) activity appears central to the "toxin-platelet-AMR" pathway driving deleterious thrombocytopenia in SA bloodstream infection, we considered the possibility that pharmacological sialidase inhibition could be of therapeutic benefit. Oseltamivir (Tamiflu ${ }^{\circledR}$ ) is a commonly prescribed FDA-approved drug designed to target influenza sialidase (neuraminidase) and lessen the severity of flu symptoms. However, oseltamivir has a degree of non-selectivity in its sialidase inhibition, as the drug was recently recognized to raise platelet counts in mice with anti-GPIb $\alpha$-mediated thrombocytopenia (43). Using Asgr2/-- mice to prevent immediate clearance of desialylated platelets, we confirmed that oseltamivir inhibits platelet desialylation in vivo during SA infection (Fig. 6C). Then using WT mice, we showed that oseltamivir significantly reduced the degree of $\alpha$-toxin-induced thrombocytopenia during WT SA infection (Fig. 6D), and both oseltamivir and established human Neu1selective sialidase inhibitor C9-butyl-amide-2-deoxy-2,3-dehydro-N-acetylneuraminic acid (DANA), significantly improved survival outcomes in lethal SA bacteremia (Fig. 6E).

Finally, to support all elements of the elucidated "toxin-platelet-AMR" pathway, we repeated several key in vivo experiments (the ticagrelor, oseltamivir and asialofetuin treatment studies as well as challenge of Asgr $^{-/-}$mice) with the SA $\Delta$ Hla knockout mutant. Given the attenuated virulence of this mutant, establishment of bacteremia in the murine IV model required an 8-fold higher challenge inoculum. Blood harvested $4 \mathrm{~h}$ post infection in all the experiments showed that the platelet count drop associated with WT 
infection was not seen in $\Delta$ Hla-infected mice in any of the models, even at the 8 -fold higher bacterial challenge inoculum (fig. S9A). Likewise, equal CFU counts of the $\Delta$ Hla knockout mutant SA were recovered in the kidney, liver, spleen, and blood of control mice vs. mice treated with either TICA, asialofetuin, or oseltamivir, as well as WT mice when compared to Asgr2 $2^{-/-}$animals (fig. S9B). As predicted by the model, deletion of $\alpha$-toxin "phenocopies" the therapeutic benefits of the drug and genetic interventions to support platelet defense against SA bloodstream infection.

\section{DISCUSSION}

SA is a leading agent of human bloodstream infection, with higher morbidity and mortality rates than other common bacterial pathogens. Successful treatment of SA bacteremia remains vexing, as the pathogen deploys diverse mechanisms for resistance to immune and antibiotic clearance. Life-threatening complications of SA bacteremia, such as metastatic infections, infective endocarditis, and disseminated intravascular coagulation, drive worsened patient outcomes. Thrombocytopenia (platelet count $<150,000 / \mathrm{mm}^{3}$ blood) is a common phenotype observed during bacteremia and is the most predictive independent risk factor for bacteremia-associated mortality, especially in cases of neonatal sepsis and critically ill septic patients in the intensive care unit $(44,45)$. While the underlying cause of thrombocytopenia is multifactorial, our mechanistic analysis of platelet-mediated defense establishes a central pathophysiologic framework aggravating platelet depletion during SA bacteremia. We show that for SA to evade platelet microbicidal activity, the pathogen deploys the cytotoxic $\alpha$-toxin, which injures platelets and stimulates the release of endogenous sialidase, thereby dysregulating the platelet clearance mechanism involving the hepatic AMR. Pharmacological targeting of multiple levels of this "toxin-plateletAMR" pathway reveal new strategies to mitigate the progression of this immunocompromised state and protect against lethal SA bacteremia (Fig. 7). 
Sialidase transfer from lysosomal compartments to the platelet cell surface may potentially be elicited by multiple surface-bound receptors including but not limited to: P2Y12, PAR-1, and PAR-4 (46). These receptors have converging intracellular signaling pathways, and studies indicate that P2Y12 functions in crosstalk with PAR receptors (47). Ticagrelor, a commonly prescribed FDA-approved P2Y12 inhibitor, hastened clearance of SA bacteremia in vivo and enhanced human platelet killing of SA ex vivo. Here we describe new effects of the drug in reducing SA $\alpha$-toxin-mediated platelet cytotoxicity, inhibiting activation of endogenous platelet sialidase activity, and preventing AMR-dependent platelet clearance. As thrombocytopenia was not observed in SA-challenged mice lacking the AMR, wholescale SA-induced platelet damage, and ticagrelor's ability to counteract this injury, may be a phenomenon unique to the high bacterial concentrations and close platelet contact present in our in vitro assays, perhaps only relevant in vivo within an infected thrombus. Conceivably, ticagrelor's primary therapeutic indication for acute coronary syndrome, reduction of platelet aggregation, may further provide protective benefit in SA bacteremia, since the pathogen produces two clotting factors, coagulase (Coa) and von Willebrand factor binding protein (vWbp), that contribute to abscess formation and systemic virulence (48). Of note, one prior report linked in vitro $\mathrm{P} 2 \mathrm{Y} 12$ activation to the release of platelet antimicrobial peptides active against SA (49). This paradoxical result may in part reflect the particular SA strain (ISP479C) used in the study, which harbors a chromosomal Tn551 insertion with a pleiotropic effect on several extracellular and cell wall proteins, including elimination of measurable $\alpha$-toxin activity (50). Platelet release of antimicrobial peptides active against SA is also activated by additional pathways, including thrombin-mediated enzymatic activation of cell surface protease-activated receptor-1 (PAR-1) still operative during P2Y12 blockade (12). The feasibility of ticagrelor as an adjunctive therapy for SA bacteremia in complex ICU patients is likely enhanced by its reversible binding to the P2Y12 receptor binding providing a very rapid onset and offset of action (51). 
Oseltamivir, a commonly used FDA-approved influenza sialidase inhibitor, maintained platelet sialylation levels to delay AMR-dependent platelet clearance, and thus provided protection against mortality in SA bloodstream infection. While one enzymatic study suggested that oseltamivir had only limited inhibitory activity against human sialidases (52), humans prescribed oseltamivir show higher platelet counts than matched controls (independent of proven influenza, (53)), and two independent case studies report successful use of the drug to restore platelet counts in a patient with immune thrombocytopenia $(54,55)$. Bacterial co-infection is estimated to have contributed to nearly all influenza deaths in the 1918 influenza pandemic and up to one-third of 2009 pandemic influenza A(H1N1) infections managed in ICUs worldwide (56). In particular, the potential for lethal synergism between SA and influenza virus has recently been documented in U.S. clinical epidemiologic studies of adult and pediatric patients $(57,58)$. In laboratoryconfirmed influenza, an inverse relationship between virus load and platelet count is seen, and viral-induced thrombocytopenia can be recapitulated in the ferret model (59). We speculate that the "two-hit" scenario of influenza neuraminidase on top of $\alpha$-toxin-induced endogenous sialidase activation may accelerate platelet clearance, depleting the host of a critical frontline defense against SA bloodstream dissemination, thus increasing the odds of fatal outcome.

An important limitation of pharmacological targeting AMR-dependent clearance of desialylated platelets to treat bacteremia is its dependency on sensitivity of the offending pathogen to platelet antimicrobial activity. A definitive or strong presumptive microbiologic diagnosis of SA would be required, precluding its use as empiric therapy wherein other pathogens, such as platelet-resistant $S$. pneumoniae, could yield adverse results (41). Multiple pathogenic mechanisms contribute to sepsis and intrinsic host factors can have differing roles depending on the pathogen involved (60). In this case, AMR function may serve protective and disadvantageous roles depending upon the pathogen and the balance of platelet action in thrombosis vs. antimicrobial activity. Prior research indicates that loss of AMR can increase platelet count and regulate thrombopoietin production (61). Mechanisms of physiologic platelet turnover remain to be 
fully established and are likely to contribute to therapeutic modulation in the future. However, it is unlikely that either ticagrelor or oseltamivir administered late in the course of severe SA-induced thrombocytopenia could quickly restore platelet counts. There, perhaps platelet transfusion could augment anti-SA killing capacity in blood, wherein the pharmacological agents could mitigate against further $\alpha$-toxin driven accelerated desialylation and AMR clearance of the donor platelets. The pathological process can also be targeted upstream at the level of the inciting SA $\alpha$-toxin, where important research on neutralizing antibodies (e.g. Medimmune 4893) and receptor antagonists (e.g. GI254023X)) have shown promising results $(14,62)$.

Therapeutic drug repurposing is an important avenue of exploration to improve clinical outcomes in serious infections where high rates of treatment failure and antibiotic resistance jeopardize patients. Elucidation of sialidase-dependent platelet homeostasis as a key battleground in host defense against SA bloodstream infection revealed the potential utility of P2Y12 and sialidase inhibition as adjunctive agents to antibiotic treatment and ICU supportive care for the critically ill. The most effective physiological concentrations to inhibit platelet cytotoxicity, sialidase activity and protect against SA bacteremia in humans are currently unknown. As FDA-approved drugs with excellent safety profiles in each class are readily at hand, we hope that carefully designed clinical investigation to validate or refute our experimental observations may follow.

\section{MATERIALS AND METHODS}

\section{Study Design}

The objective of this study was to understand the mechanistic basis of platelet homeostasis and function during SA bacteremia to guide future therapeutic approaches. Our analysis of patient data and SA isolates from a published 2009-10 IRB-approved study (25) of SA bacteremia at the University of Wisconsin Hospital linked thrombocytopenia to patient mortality and elevated $\alpha$-toxin production. Both correlations 
were corroborated in a UC San Diego IACUC-approved murine model of SA bacteremia. UC San Diego IRB-approved ex vivo studies with freshly isolated human platelets were used to show the FDA-approved P2Y12 antagonist ticagrelor blocked $\alpha$-toxin induced platelet injury and sialidase activation, improving microbial killing. Infection studies WT and isogenic AMR-deficient mice were used to link $\alpha$-toxin mediated platelet sialidase activation to accelerated thrombocytopenia and impaired SA clearance, which could be counteracted by ticagrelor or the FDA-approved sialidase inhibitor oseltamivir.

\section{Statistical Analysis}

All in vitro, ex vivo, and in vivo data were collected from three or more $(\geq 3)$ independent experiments with $\geq 3$ biological replicates and are represented as mean \pm standard error mean (SEM), unless otherwise stated. For descriptive data (transmission electron microscopy and histopathologic staining), experiments were performed at least twice independently with $\geq 3$ biological replicates and illustrated as best representative images. The alpha level used for all tests was 0.05; unpaired Student's t-test, one-way ANOVA with Bonferroni's multiple comparisons test or two-way ANOVA with Bonferroni's multiple comparisons test was performed as explained in figure legends to determine statistical significance. For comparison of survival curves, a log-rank (Mantel-Cox) test was performed. Statistical analyses were done using GraphPad Prism, version 8.42 (GraphPad Software Inc., La Jolla, CA, USA). P values $<0.05$ were considered statistically significant. 


\section{SUPPLEMENTARY MATERIALS}

Materials and Methods

Fig. S1. Thrombocytopenia established by anti-CD41 antibody treatment of mice.

Fig. S2. Grouping of $\alpha$-toxin expression of SA isolates from bacteremia patients.

Fig. S3. Generation of an isogenic SA $\Delta$ Hla mutant.

Fig. S4. Exclusion of ticagrelor off-target effects on SA immune cell interactions and growth.

Fig. S5. Additional effects of SA or TICA on human platelet phenotypes in vitro.

Fig. S6. Effects of SA challenge on platelets and thrombopoiesis in vivo

Fig. S7. Organ pathology in murine SA infection with or without ticagrelor treatment.

Fig. S8. Neu1 is predominantly detected on the platelet cell surface and is induced by SA exposure Fig. S9. SA $\alpha$-toxin deletion mutant phenocopies protective effects of TICA, oseltamivir and AMR loss/inhibition.

References (63-69)

\section{ACKNOWLEDGEMENTS}

This work was supported by NIH grants HL125352 (JDM, VN), HL107150 (VN), KD048247 (JDM), AI124326 (GS, VN), HD090259 (GS, VN) and AI13262 (WR). JS was supported the UCSD PharmD/PhD Program and IC by the UCSD Research Training Program for Veterinarians (NIH OD017863). The authors thank Ajit Varki for his critical insights on human and murine sialobiology. 


\section{REFERENCES}

1. K. B. Laupland, Incidence of bloodstream infection: a review of population-based studies, Clin. Microbiol. Infect. 19, 492-500 (2013).

2. A. J. Kaasch, G. Barlow, J. D. Edgeworth, V. G. Fowler Jr, M. Hellmich, S. Hopkins, W. V. Kern, M. J. Llewelyn, S. Rieg, J. Rodriguez-Baño, M. Scarborough, H. Seifert, A. Soriano, R. Tilley, M. E. Tőrők, V. Weiß, A. P. R. Wilson, G. E. Thwaites, ISAC, INSTINCT, SABG, UKCIRG, and Colleagues, Staphylococcus aureus bloodstream infection: a pooled analysis of five prospective, observational studies, J. Infect. 68, 242-251 (2014).

3. A. Del Rio, C. Cervera, A. Moreno, Patients at risk of complications of Staphylococcus aureus bloodstream infection, Clin. Infect. Dis. 48 Suppl 4:S246-53 (2009)

4. E.-J. Joo, D. A. Park, C.-I. Kang, D. R. Chung, J.-H. Song, S. M. Lee, K. R. Peck, Reevaluation of the impact of methicillin-resistance on outcomes in patients with Staphylococcus aureus bacteremia and endocarditis, Korean J. Intern. Med. 34, 1347-1362 (2019).

5. L. Thomer, O. Schneewind, D. Missiakas, Pathogenesis of Staphylococcus aureus bloodstream infections, Ann. Rev. Pathol. 11, 343-364 (2016).

6. A. Laarman, F. Milder, J. van Strijp, S. Rooijakkers, Complement inhibition by Gram-positive pathogens: molecular mechanisms and therapeutic implications, J. Mol. Med. 88, 115-120 (2010).

7. H. K. Kim, V. Thammavongsa, O. Schneewind, D. Missiakas, Recurrent infections and immune evasion strategies of Staphylococcus aureus, Curr. Opin. Microbiol. 15, 92-99 (2012).

8. W. McGuinness, S. Kobayashi, F. DeLeo, Evasion of neutrophil killing by Staphylococcus aureus, Pathogens 5, 32 (2016).

9. M. R. Yeaman, Platelets: at the nexus of antimicrobial defence, Nat. Rev. Microbiol. 12, 426-437 (2014).

10. F. Gaertner, Z. Ahmad, G. Rosenberger, S. Fan, L. Nicolai, B. Busch, G. Yavuz, M. Luckner, H. IshikawaAnkerhold, R. Hennel, A. Benechet, M. Lorenz, S. Chandraratne, I. Schubert, S. Helmer, B. Striednig, K. Stark, M. Janko, R. T. Böttcher, A. Verschoor, C. Leon, C. Gachet, T. Gudermann, M. M. y. Schnitzler, Z. Pincus, M. Iannacone, R. Haas, G. Wanner, K. Lauber, M. Sixt, S. Massberg, Migrating platelets are mechano-scavengers that collect and bundle bacteria, Cell 171, 1368-1382.e23 (2017).

11. S.-J. Kim, C. N. Jenne, Role of platelets in neutrophil extracellular trap (NET) production and tissue injury, Semin. Immunol. 28, 546-554 (2016).

12. R. A. Ali, L. M. Wuescher, K. R. Dona, R. G. Worth, Platelets Mediate Host Defense against Staphylococcus aureus through direct bactericidal activity and by enhancing macrophage activities, J. Immunol. 198, 344-351 (2017).

13. C. H. Y. Wong, C. N. Jenne, B. Petri, N. L. Chrobok, P. Kubes, Nucleation of platelets with blood-borne pathogens on Kupffer cells precedes other innate immunity and contributes to bacterial clearance, Nat. Immunol. 14, 785-792 (2013).

14. B. G. J. Surewaard, A. Thanabalasuriar, Z. Zeng, C. Tkaczyk, T. S. Cohen, B. W. Bardoel, S. K. Jorch, C. Deppermann, J. B. Wardenburg, R. P. Davis, C. N. Jenne, K. C. Stover, B. R. Sellman, P. Kubes, $\alpha$-Toxin induces platelet aggregation and liver injury during Staphylococcus aureus sepsis, Cell Host Microbe 24, 271-284.e3 (2018).

15. F. Cognasse, K. A. Nguyen, P. Damien, A. McNicol, B. Pozzetto, H. Hamzeh-Cognasse, O. Garraud, The 
inflammatory role of platelets via their TLRs and Siglec receptors, Front. Immunol. 6, 83 (2015).

16. A.-S. Anabel, P.-C. Eduardo, H.-C. P. Antonio, S.-M. Carlos, N.-M. Juana, T.-A. Honorio, V.-S. Nicolás, A.-R. S. Roberto, Human platelets express Toll-like receptor 3 and respond to poly I:C, Hum. Immunol., 75, 1244-1251 (2014).

17. B. F. Kraemer, R. A. Campbell, H. Schwertz, M. J. Cody, Z. Franks, N. D. Tolley, W. H. A. Kahr, S. Lindemann, P. Seizer, C. C. Yost, G. A. Zimmerman, A. S. Weyrich, Novel anti-bacterial activities of $\beta$-defensin 1 in human platelets: suppression of pathogen growth and signaling of neutrophil extracellular trap formation, PLoS Pathog. 7, e1002355 (2011).

18. M. R. Yeaman, D. C. Norman, A. S. Bayer, Staphylococcus aureus susceptibility to thrombin-induced platelet microbicidal protein is independent of platelet adherence and aggregation in vitro, Infect. Immun. 60, 2368-2374 (1992).

19. M. Wolff, S. Handtke, R. Palankar, J. Wesche, T. P. Kohler, C. Kohler, Y. Gruel, S. Hammerschmidt, A. Greinacher, Activated platelets kill Staphylococcus aureus, but not Streptococcus pneumoniae-The role of Fc $\gamma$ RIIa and platelet factor 4/heparin antibodies, J. Thromb. Haemost. 18, 1459-1468 (2020).

20. M. E. Powers, R. E. N. Becker, A. Sailer, J. R. Turner, J. B. Wardenburg, Synergistic action of Staphylococcus aureus $\alpha$-toxin on platelets and myeloid lineage cells contributes to lethal sepsis, Cell Host Microbe 17, 775-787 (2015).

21. J. R. Fitzgerald, A. Loughman, F. Keane, M. Brennan, M. Knobel, J. Higgins, L. Visai, P. Speziale, D. Cox, T. J. Foster, Fibronectin-binding proteins of Staphylococcus aureus mediate activation of human platelets via fibrinogen and fibronectin bridges to integrin GPIIb/IIIa and IgG binding to the FcgammaRIIa receptor, Mol. Microbiol. 59, 212-230 (2006).

22. A. Loughman, J. R. Fitzgerald, M. P. Brennan, J. Higgins, R. Downer, D. Cox, T. J. Foster, Roles for fibrinogen, immunoglobulin and complement in platelet activation promoted by Staphylococcus aureus clumping factor A, Mol. Microbiol. 57, 804-818 (2005).

23. L. O’Brien, S. W. Kerrigan, G. Kaw, M. Hogan, J. Penadés, D. Litt, D. J. Fitzgerald, T. J. Foster, D. Cox, Multiple mechanisms for the activation of human platelet aggregation by Staphylococcus aureus: roles for the clumping factors ClfA and ClfB, the serine-aspartate repeat protein SdrE and protein A. Mol. Microbiol. 44, 1033-1044 (2002).

24. L. M. Wuescher, A. Takashima, R. G. Worth, A novel conditional platelet depletion mouse model reveals the importance of platelets in protection against Staphylococcus aureus bacteremia J. Thrombos. Haemostas. 13, 303$313(2015)$.

25. W. E. Rose, J. C. Eickhoff, S. K. Shukla, M. Pantrangi, S. Rooijakkers, S. E. Cosgrove, V. Nizet, G. Sakoulas, Elevated serum interleukin-10 at time of hospital admission is predictive of mortality in patients with Staphylococcus aureus bacteremia, J. Infect. Dis. 206, 1604-1611 (2012).

26. A. Gafter-Gvili, N. Mansur, A. Bivas, N. Zemer-Wassercug, J. Bishara, L. Leibovici, M. Paul, Thrombocytopenia in Staphylococcus aureus bacteremia: risk factors and prognostic importance, Mayo Clinic Proc., 86, 389-396 (2011).

27. L. L. Peters, E. M. Cheever, H. R. Ellis, P. A. Magnani, K. L. Svenson, R. Von Smith, M. A. Bogue, Large-scale, high-throughput screening for coagulation and hematologic phenotypes in mice Physiol. Genom. 11, 185-193 (2002).

28. S. Bhakdi, M. Muhly, U. Mannhardt, F. Hugo, K. Klapettek, C. Mueller-Eckhardt, L. Roka, Staphylococcal $\alpha$ toxin promotes blood coagulation via attack on human platelets, J. Exp. Med., 168, 527-542 (1988).

29. P. Damman, P. Woudstra, W. J. Kuijt, R. J. de Winter, S. K. James, P2Y12 platelet inhibition in clinical practice, J. Thrombos. Thrombolys. 33, 143-153 (2012). 
30. J. Winning, J. Reichel, Y. Eisenhut, J. Hamacher, M. Kohl, H. P. Deigner, R. A. Claus, M. Bauer, W. Lösche, Anti-platelet drugs and outcome in severe infection: Clinical impact and underlying mechanisms, Platelets 20, 5057 (2009).

31. J. Pepin, V. Tremblay, D. Bechard, F. Rodier, C. Walker, D. Dufresne, A. Lafontaine, N. Li, C. Lacroix, L. Lanthier, Chronic antiplatelet therapy and mortality among patients with infective endocarditis, Clin. Microbiol. Infect. 15, 193-199 (2009).

32. M. Osthoff, J. A. Sidler, B. Lakatos, R. Frei, M. Dangel, M. Weisser, M. Battegay, A. F. Widmer, Low-dose acetylsalicylic acid treatment and impact on short-term mortality in Staphylococcus aureus bloodstream infection: a propensity score-matched cohort study, Crit. Care Med. 44, 773 (2016).

33. J. Trauer, S. Muhi, E. S. McBryde, S. A. Al Harbi, Y. M. Arabi, A. J. Boyle, R. Cartin-Ceba, W. Chen, Y.-T. Chen, M. Falcone, O. Gajic, J. Godsell, M. N. Gong, D. Kor, W. Lösche, D. F. McAuley, H. R. O’Neal Jr, M. Osthoff, G. P. Otto, M. Sossdorf, M.-J. Tsai, J. C. Valerio-Rojas, T. van der Poll, F. Violi, L. Ware, A. F. Widmer, M. A. Wiewel, J. Winning, D. P. Eisen, Quantifying the effects of prior acetyl-salicylic acid on sepsis-related deaths: an individual patient data meta-analysis using propensity matching, Crit. Care Med. 45, 1871-1879 (2017).

34. J. Winning, J. Neumann, M. Kohl, R. A. Claus, K. Reinhart, M. Bauer, W. Lösche, Antiplatelet drugs and outcome in mixed admissions to an intensive care unit, Crit. Care Med. 38, 32-37 (2010).

35. K. M. Hoffmeister, H. Falet, Platelet clearance by the hepatic Ashwell-Morrell receptor: mechanisms and biological significance, Thrombos. Res., 141, S68-S72 (2016).

36. A. J. G. Jansen, A. J. Gerard Jansen, E. C. Josefsson, V. Rumjantseva, Q. P. Liu, H. Falet, W. Bergmeier, S. M. Cifuni, R. Sackstein, U. H. von Andrian, D. D. Wagner, J. H. Hartwig, K. M. Hoffmeister, Desialylation accelerates platelet clearance after refrigeration and initiates GPIb $\alpha$ metalloproteinase-mediated cleavage in mice, Blood 119, 1263-1273 (2012).

37. X.-H. Zhang, Q.-M. Wang, J.-M. Zhang, F.-E. Feng, F.-R. Wang, H. Chen, Y.-Y. Zhang, Y.-H. Chen, W. Han, L.-P. Xu, K.-Y. Liu, X.-J. Huang, Desialylation is associated with apoptosis and phagocytosis of platelets in patients with prolonged isolated thrombocytopenia after allo-HSCT, J. Hematol. Oncol., 8, 116 (2015).

38. T. J. Rink, A. Sanchez, T. J. Hallam, Diacylglycerol and phorbol ester stimulate secretion without raising cytoplasmic free calcium in human platelets, Nature 305, 317-319 (1983).

39. D. E. Knight, H. von Grafenstein, C. M. Athayde, Calcium-dependent and calcium-independent exocytosis, Trends Neurosci. 12, 451-458 (1989).

40. P. K. Grewal, S. Uchiyama, D. Ditto, N. Varki, D. T. Le, V. Nizet, J. D. Marth, The Ashwell receptor mitigates the lethal coagulopathy of sepsis, Nat. Med. 14, 648-655 (2008).

41. P. K. Grewal, P. V. Aziz, S. Uchiyama, G. R. Rubio, R. D. Lardone, D. Le, N. M. Varki, V. Nizet, J. D. Marth, Inducing host protection in pneumococcal sepsis by preactivation of the Ashwell-Morell receptor, Proc. Natl. Acad. Sci. U. S. A. 110, 20218-20223 (2013).

42. L. G. Ellies, D. Ditto, G. G. Levy, M. Wahrenbrock, D. Ginsburg, A. Varki, D. T. Le, J. D. Marth, Sialyltransferase ST3Gal-IV operates as a dominant modifier of hemostasis by concealing asialoglycoprotein receptor ligands, Proc. Natl. Acad. Sci. U. S. A. 99, 10042-10047 (2002).

43. J. Li, D. E. van der Wal, G. Zhu, M. Xu, I. Yougbare, L. Ma, B. Vadasz, N. Carrim, R. Grozovsky, M. Ruan, L. Zhu, Q. Zeng, L. Tao, Z.-M. Zhai, J. Peng, M. Hou, V. Leytin, J. Freedman, K. M. Hoffmeister, H. Ni, Desialylation is a mechanism of $\mathrm{Fc}$-independent platelet clearance and a therapeutic target in immune thrombocytopenia, Nat. Commun. 6 (2015), 6:7737.

44. I. M. C. Ree, S. F. Fustolo-Gunnink, V. Bekker, K. J. Fijnvandraat, S. J. Steggerda, E. Lopriore, 
Thrombocytopenia in neonatal sepsis: Incidence, severity and risk factors, PLoS One 12, e0185581 (2017).

45. T. A. M. Claushuis, L. A. van Vught, B. P. Scicluna, M. A. Wiewel, P. M. Klein, A. J. Hoogendijk, D. S. Y. Ong, O. L. Cremer, J. Horn, M. Franitza, M. R. Toliat, P. Nürnberg, A. H. Zwinderman, M. J. Bonten, M. J. Schultz, T. van der Poll, Thrombocytopenia is associated with a dysregulated host response in critically ill sepsis patients, Blood 127, 3062-3072 (2016).

46. A. L. Södergren, A.-C. B. Svensson Holm, S. Ramström, E. G. Lindström, M. Grenegård, K. Öllinger, Thrombininduced lysosomal exocytosis in human platelets is dependent on secondary activation by ADP and regulated by endothelial-derived substances, Platelets 27, 86-92 (2016).

47. T. H. Smith, J. G. Li, M. R. Dores, J. Trejo, Protease-activated receptor-4 and purinergic receptor P2Y12 dimerize, co-internalize, and activate Akt signaling via endosomal recruitment of $\beta$-arrestin. J. Biol. CHem. 292, 13867-13878 (2017).

48. A. G. Cheng, M. McAdow, H. K. Kim, T. Bae, D. M. Missiakas, O. Schneewind, Contribution of coagulases towards Staphylococcus aureus disease and protective immunity, PLoS Pathog. 6, e1001036 (2010).

49. D. A. Trier, K. D. Gank, D. Kupferwasser, N. Y. Yount, W. J. French, A. D. Michelson, L. I. Kupferwasser, Y. Q. Xiong, A. S. Bayer, M. R. Yeaman, Platelet antistaphylococcal responses occur through P2X1 and P2Y12 receptor-induced activation and kinocidin release Infect. Immun. 76, 5706-5713 (2008).

50. A. L. Cheung, C. Wolz, M. R. Yeaman, A. S. Bayer, Insertional inactivation of a chromosomal locus that modulates expression of potential virulence determinants in Staphylococcus aureus, , I J. Bacteriol. 177, 3220-3226 (1995).

51. D. Goel, Ticagrelor: The first approved reversible oral antiplatelet agent Int. J. Appl. Basic Med. Res. 3, 19 (2013).

52. K. Hata, K. Koseki, K. Yamaguchi, S. Moriya, Y. Suzuki, S. Yingsakmongkon, G. Hirai, M. Sodeoka, M. von Itzstein, T. Miyagi, Limited inhibitory effects of oseltamivir and zanamivir on human sialidases Antimicrob. Agents Chemother. 52, 3484-3491 (2008).

53. A. J. G. Jansen, J. Peng, H.-G. Zhao, M. Hou, H. Ni, Sialidase inhibition to increase platelet counts: A new treatment option for thrombocytopenia Am. J. Hematol. 90, E94-E95 (2015).

54. P. Bigot, M. Auffret, S. Gautier, M. Weinborn, N.-K. Ettahar, P. Coupé, Unexpected platelets elevation in a patient with idiopathic thrombocytopenia treated with oseltamivir for influenza infection Fundamen. \& Clin. Pharmacol. 30, 483-485 (2016).

55. B. Alioglu, A. Tasar, C. Ozen, B. Selver, Y. Dallar, An experience of oseltamivir phosphate (Tamiflu $\left.{ }^{\mathrm{tm}}\right)$ in a pediatric patient with chronic idiopathic thrombocytopenic purpura: a case report Pathophys. Haemostas. Thrombos. 37, 55-58 (2009).

56. D. S. Chertow, M. J. Memoli, Bacterial coinfection in influenza, JAMA 309, 275 (2013).

57. J. S. McDanel, E. N. Perencevich, J. Storm, D. J. Diekema, L. Herwaldt, J. Kristie Johnson, P. L. Winokur, M. L. Schweizer, Increased mortality rates associated with Staphylococcus aureus and influenza co-infection, Maryland and Iowa, USA, Emerg. Infect. Dis. 22, 1253-1256 (2016).

58. L. Finelli, A. Fiore, R. Dhara, L. Brammer, D. K. Shay, L. Kamimoto, A. Fry, J. Hageman, R. Gorwitz, J. Bresee, T. Uyeki, Influenza-associated pediatric mortality in the United States: increase of Staphylococcus aureus coinfection, Pediatrics 122, 805-811 (2008).

59. A. J. G. Jansen, A. J. Gerard Jansen, H. Z. Low, J. van den Brand, D. van Riel, A. Osterhaus, E. van der Vries, Platelets can phagocytose influenza virus which may contribute to the occurrence of thrombocytopenia during influenza infection, Blood 128, 1358-1358 (2016). 
60. W. H. Yang, D. M. Heithoff, P. V. Aziz, B. Haslund-Gourley, J. S. Westman, S. Narisawa, A. B. Pinkerton, J. L. Millán, V. Nizet, M. J. Mahan, J. D. Marth, Accelerated aging and clearance of host anti-inflammatory enzymes by discrete pathogens fuels sepsis, Cell Host Microbe 24, 500-513.e5 (2018).

61. R. Grozovsky, A. J. Begonja, K. Liu, G. Visner, J. H. Hartwig, H. Falet, K. M. Hoffmeister, The Ashwell-Morell receptor regulates hepatic thrombopoietin production via JAK2-STAT3 signaling, Nat. Med. 21, 47-54 (2015).

62. G. R. Sampedro, A. C. DeDent, R. E. N. Becker, B. J. Berube, M. J. Gebhardt, H. Cao, J. B. Wardenburg, Targeting Staphylococcus aureus $\alpha$-toxin as a novel approach to reduce severity of recurrent skin and soft-tissue infections, J. Infect. Dis. 210, 1012-1018 (2014). 
A

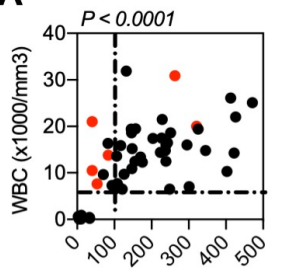

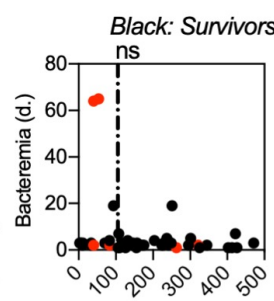

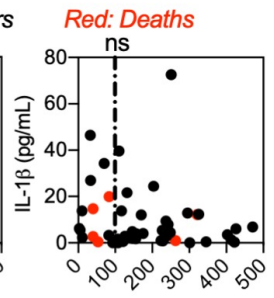

Platelets $\left(x 1000 / \mathrm{mm}^{3}\right)$

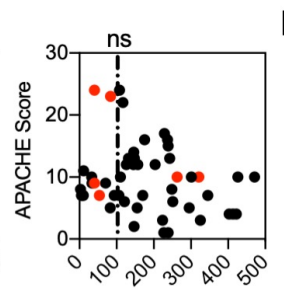

$\mathbf{B}$

\begin{tabular}{|c|c|c|c|}
\hline \multicolumn{2}{|c|}{ Black: Survivors } & \multicolumn{2}{|c|}{ Red: Deaths } \\
\hline Group & Yes & No & $x^{2}$ test \\
\hline$W B C<5 K$ & 610 & 3716 & $P=0.32$ \\
\hline$W B C<10 K$ & $8 \mid 1$ & 3515 & $P=0.90$ \\
\hline$W B C<20 K$ & 512 & 3814 & $P=0.15$ \\
\hline $\mathrm{PLTS}<100 \mathrm{~K}$ & 914 & 3412 & $P=.017$ \\
\hline
\end{tabular}

C

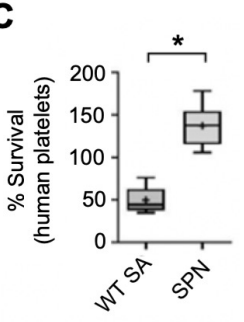

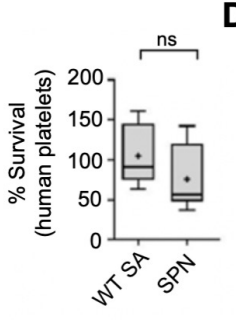

D

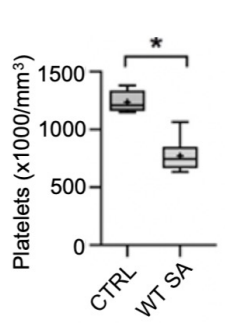

E

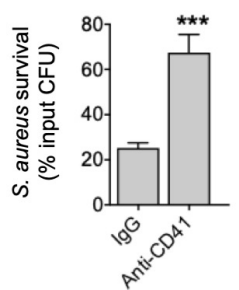

$\mathbf{F}$

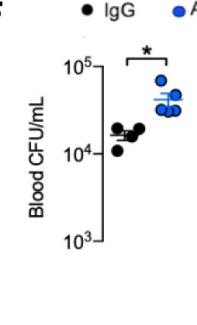

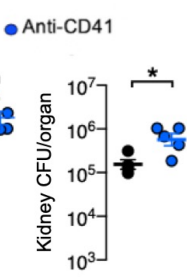
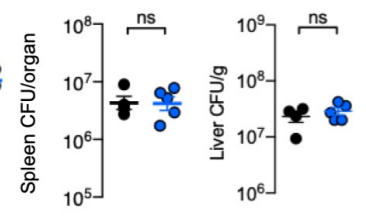

G

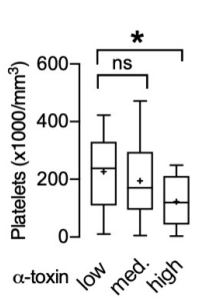

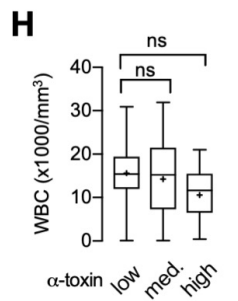

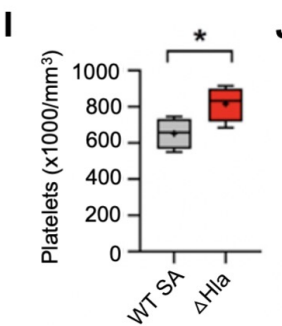

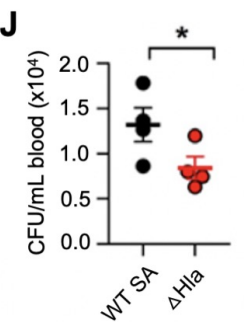

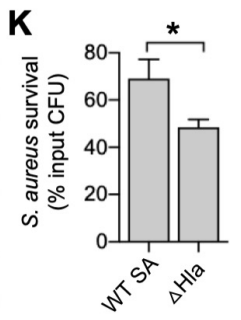

Fig. 1. Platelets are essential for blood immunity against Staphylococcus aureus (SA) bacteremia and SA $\alpha$ toxin induces thrombocytopenia to evade platelet-mediated microbicidal activity. (A) Correlation of circulating platelet counts with leukocyte counts and duration of bacteremia in 49 consecutive patients with SA bacteremia from a tertiary medical center; Spearman's rank correlation coefficient compared variables. (B) Mortality in patient cohort associated with different leukocyte and platelet count cutoffs; Chi-square without Yates correction, $\mathrm{P}<0.05$ considered significant. (C) Washed isolated human platelets and neutrophils exposed to SA or SPN at MOI $=0.01$ for $2 \mathrm{~h}$. Samples sonicated, serial diluted, and plated on THA plates for enumeration of bacterial colony forming units (CFU). (D) Reduction in platelet count $2 \mathrm{~h}$ post intravenous infection of mice with SA $(\mathrm{n}=8)$ vs. non-infected littermate control $(\mathrm{n}=4)$. Experiment reproduced $\mathrm{x} 2$ and data pooled; data represented as mean \pm SEM. (E) Ex vivo killing of SA upon $2 \mathrm{~h}$ co-incubation with blood collected from mice $16 \mathrm{~h}$ after treatment with anti-CD41 antibody $(\mathrm{n}=9)$ or IgG control $(\mathrm{n}=12)$. (F) Mice treated with platelet-depleting anti-CD41 antibody $(\mathrm{n}=5)$ or IgG control $(\mathrm{n}$ =4) for $16 \mathrm{~h}$ prior to intravenous SA infection. Organs harvested and CFU enumerated $2 \mathrm{~h}$ post-infection in triplicate for each sample. (G) Assessment of $\alpha$-toxin production by the infecting SA isolate in 49 consecutive bacteremia cases and its association with patient platelet counts and $(\mathbf{H})$ white blood cell counts. (I) Wild-type SA $(\mathrm{n}=4)$ or isogenic $\triangle$ Hla mutant $(\mathrm{n}=4)$ intravenously challenged outbred CD-1 mice. Blood harvested by cardiac puncture and CBC obtained 4 h post-infection. (J) Wild-type SA $(n=4)$ or isogenic $\Delta$ Hla mutant $(n=4)$ intravenously challenged outbred CD-1 mice. Blood harvested by cardiac puncture and enumeration of colony forming units (CFU) 4 hours post-intravenous infection. (K) Ex vivo killing of SA by freshly isolated human platelets ( $2 \mathrm{~h}$ co-incubation) vs. isogenic $\Delta$ Hla mutant. All data represented as mean \pm SEM and representative of at least 3 independent experiments. Statistical significance was determined by unpaired two-tailed Student's T-test (C-F, I, J, K) or one way analysis of variance (ANOVA) with Bonferroni's multiple comparisons test $(\mathrm{G}, \mathrm{H}) . * \mathrm{P}<0.05$. For floating bar graphs, + denotes the mean, whiskers represent min. to max, and floating box represents 25 th to 75 th percentile. 

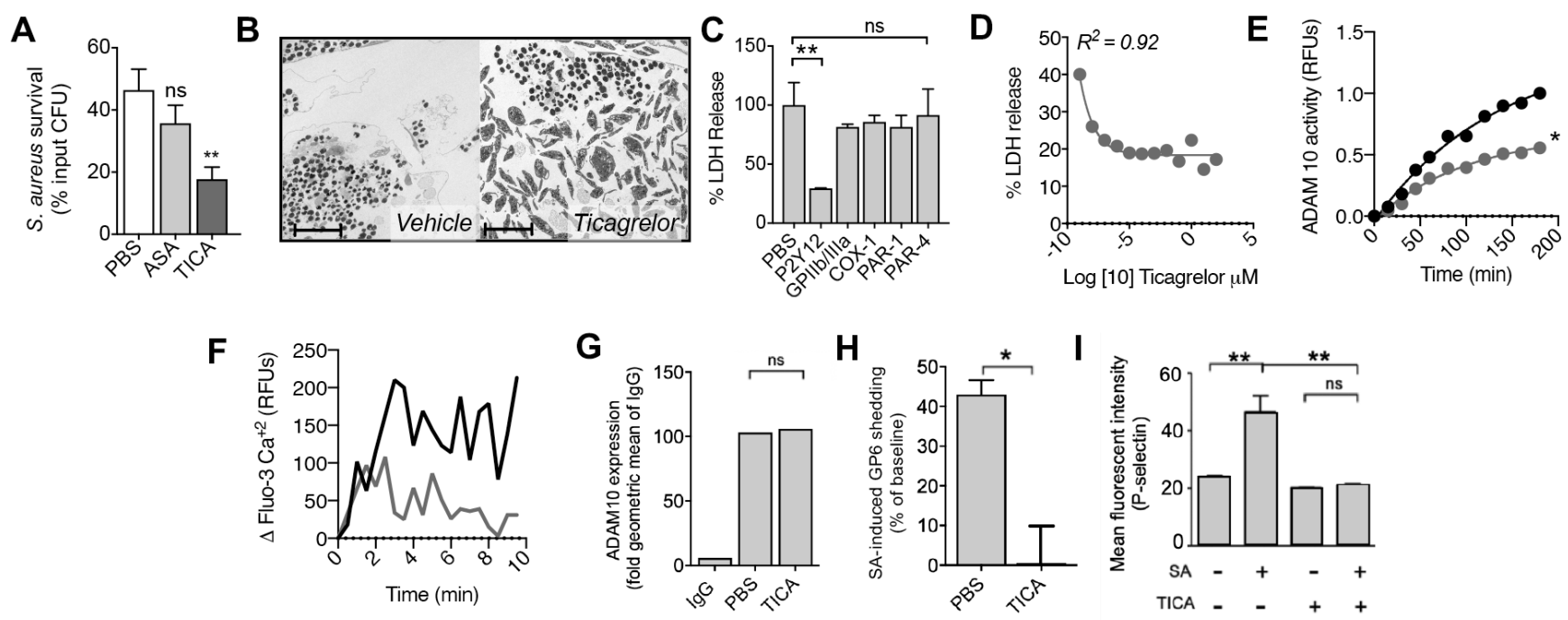

Fig. 2. FDA-approved P2Y12 inhibitor ticagrelor blocks SA $\alpha$-toxin-mediated platelet cytotoxicity. (A) Effect of $10 \mu \mathrm{M}$ aspirin (ASA) and $10 \mu \mathrm{M}$ ticagrelor (TICA) (15 min pretreatment ex vivo) on human platelet killing of MRSA for $2 \mathrm{~h}(\mathrm{n}=9)$. Experiments performed in triplicate and repeated three times. (B) Representative transmission electron microscopy image of platelets pre-treated with or without $10 \mu \mathrm{M}$ TICA and exposed to MRSA at MOI: 0.1 for $2 \mathrm{~h}$. (C) P2Y12 inhibitor (TICA) pretreatment blocks human platelet cytotoxicity by $5 \mu \mathrm{g} / \mathrm{ml}$ purified $\alpha$-toxin as measured by LDH release $(n=3)$ in a $(D)$ dose-dependent manner. Inhibitors: P2Y12 (ticagrelor), GPIIb/IIIa (eptifibatide), COX-1 (SC560), PAR-1 (vorapaxar), and PAR-4 (ML-354). (E) TICA treatment of human platelets reduces proteolytic cleavage of an ADAM10-specific fluorogenic substrate. Data representative of three independent experiments and statistical significance determined by least squares ordinary fit, $* \mathrm{P}<0.5$. (F) Measurement of intracellular calcium in human platelets loaded with $2 \mu \mathrm{M}$ Fluo-3 dye and stimulated with $5 \mu \mathrm{g} / \mathrm{mL}$ recombinant $\alpha-$ toxin; calcium influx is measured every $30 \mathrm{sec}$ by fluorescence and normalized to baseline (non-stimulated platelet control). (G) TICA treatment of human platelets did not alter surface ADAM-10 expression as determined by flow cytometry. Surface ADAM-10 expression on human platelets treated with TICA or control was measured by flow cytometry. (H) Human platelets with or without TICA treatment were infected with MRSA at MOI $=0.1$ for 90 min. Surface glycoprotein-6 (GP6) was measured by flow cytometry and \% decreased expression (GP6 shedding) calculated. (I) Human platelet P-selectin expression indicating platelet activation measured by flow cytometry with or without MRSA challenge $(\mathrm{MOI}=0.1)$ and with or without TICA aor $90 \mathrm{~min}$. All data represented as mean $\pm \mathrm{SEM}$ and are representative of at least 3 independent experiments. Statistical significance was determined by One way ANOVA with Bonferroni's multiple comparisons test (A,C,G), unpaired two-tailed Student's T-test $(\mathrm{H})$ and twoway analysis of variance (ANOVA) with Bonferroni's multiple comparisons posttest (I). ${ }^{*} \mathrm{P}<0.05, * * \mathrm{P}<0.005$. PBS, phosphate buffered saline; ns, not significant. 


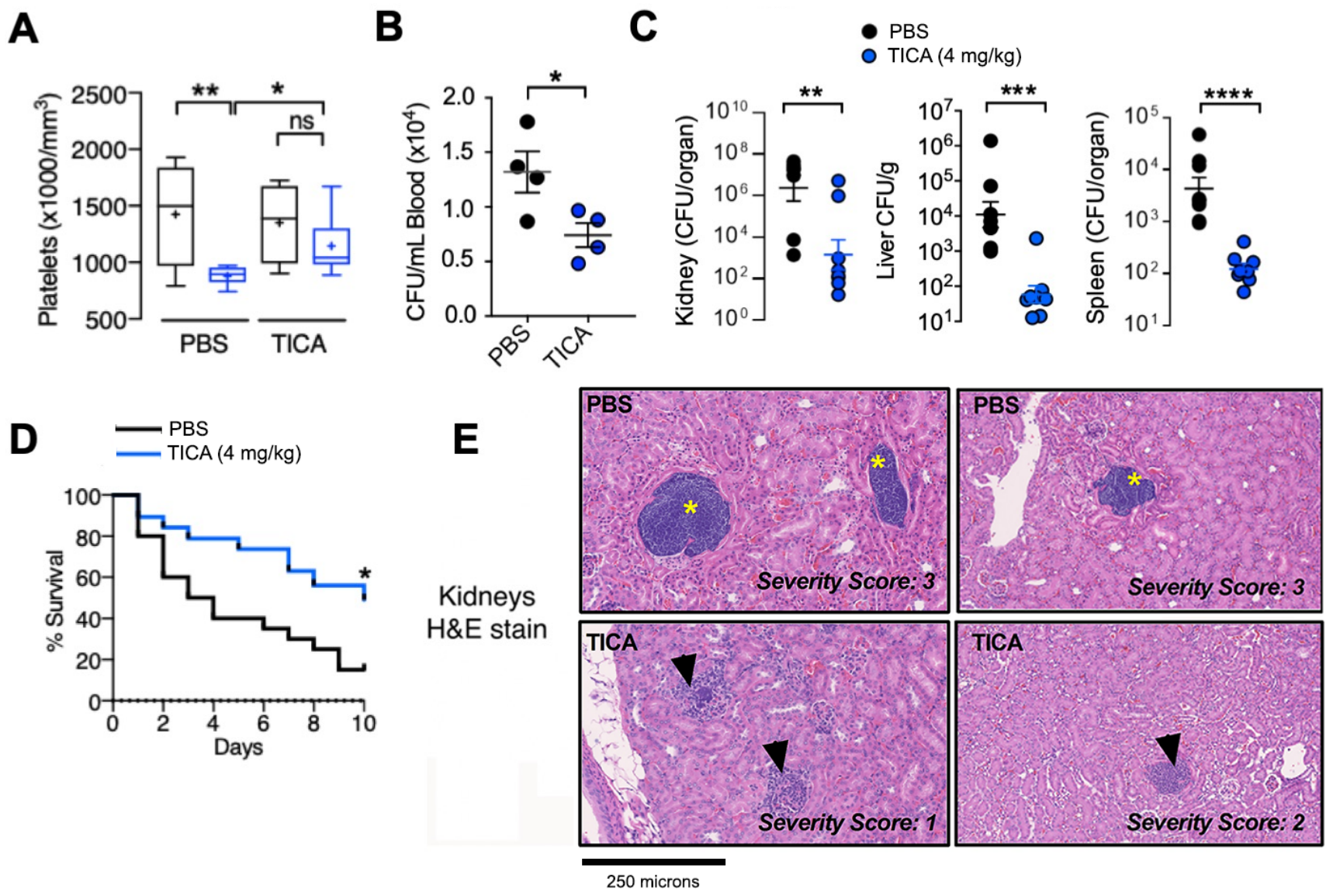

Fig. 3. FDA-approved P2Y12 inhibitor ticagrelor protects against SA bacteremia. (A and B) Blood harvested by cardiac puncture and both platelets and bacterial colony forming unit (CFU) burden enumerated from outbred CD1 mice treated with $10 \mu \mathrm{M}$ ticagrelor $(\mathrm{n}=9)$ or PBS $(\mathrm{n}=9)$ control and intravenously infected with SA. (C) Enumeration of bacterial colony forming unit (CFU) burden at $72 \mathrm{~h}$ in organs of mice pretreated with vehicle (PBS) or $4 \mathrm{mg} / \mathrm{kg}$ Ticagrelor $12 \mathrm{~h}$ prior to intravenous SA and q $12 \mathrm{~h}$ thereafter; $(\mathrm{n}=8)$. (D) Mortality curves of outbred CD1 mice pretreated with vehicle (PBS) or $4 \mathrm{mg} / \mathrm{kg}$ Ticagrelor beginning $24 \mathrm{~h}$ prior to intravenous SA infection then $\mathrm{q}$ $12 \mathrm{~h}$ over a 10-day observation period $(\mathrm{n}=20)$. Independent experiments were repeated $\mathrm{x} 2$ and data pooled. $(\mathbf{E})$ Hematoxylin and eosin stain (H\&E) of representative histological kidney sections from mice pre-treated with PBS vehicle or $4 \mathrm{mg} / \mathrm{kg}$ ticagrelor $12 \mathrm{~h}$ prior to SA infection and $\mathrm{q} 12 \mathrm{~h}$ thereafter for $72 \mathrm{~h} ;(\mathrm{n}=8)$. Yellow stars denote formation of dense bacterial colonies and black arrows represent immune infiltrate. All histological sections are representative photos of at least 6 samples per two independent experiments. Where applicable, results are represented as mean \pm SEM and statistical significance was determined by unpaired two-tailed Student's T-test (B,C), and twoway ANOVA with Bonferroni's multiple comparisons posttest (A). For survival curves, statistical significance determined by Log-rank Mantel-Cox test (D); ${ }^{*} \mathrm{P}<0.05$. For floating bar graphs, + denotes the mean, whiskers represent $\min$. to max, and floating box represents 25 th to 75 th percentile. Unless otherwise stated, $* \mathrm{P}<0.05, * * \mathrm{P}<$ $0.005, * * * \mathrm{P}<0.0005$. 
A

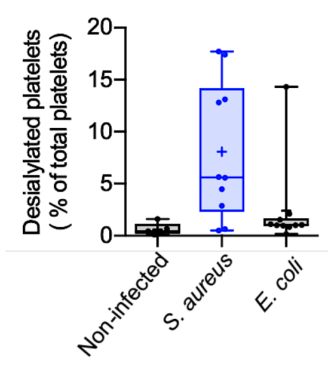

B

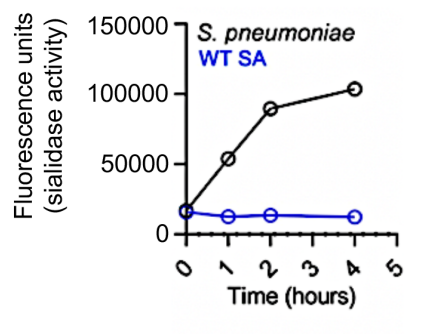

C

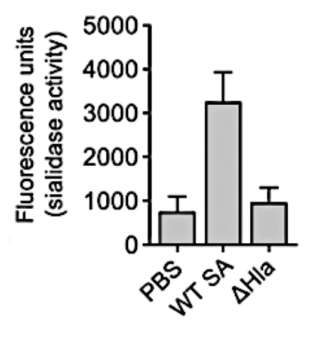

D

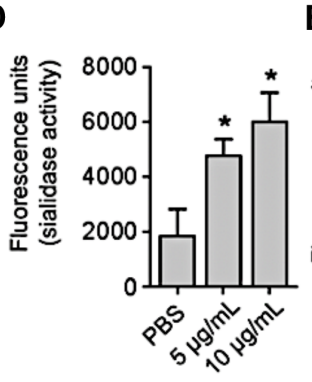

$\mathbf{E}$

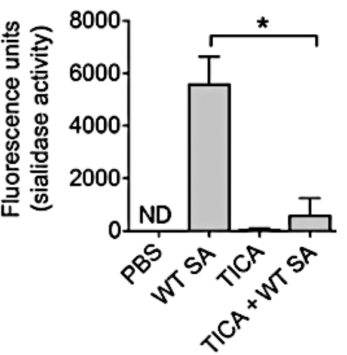

Fig. 4. SA $\alpha$-toxin activates endogenous platelet sialidase activity. (A) \% desialylated platelets in platelet rich plasma from non-infected control, SA bacteremia or Escherichia coli bacteremia patients measured by flow cytometry. (B) SA and S. pneumoniae sialidase activity assessed for over $4 \mathrm{~h}$. (C) Sialidase activity examined on washed human platelets exposed to WT SA or its isogenic $\Delta$ Hla for $1 \mathrm{~h}$ or (D) sialidase activity examined on washed human platelets exposed to $5 \mu \mathrm{g} / \mathrm{mL}$ and $10 \mu \mathrm{g} / \mathrm{mL}$ recombinant $\alpha$-toxin for $30 \mathrm{~min}$. (E) Sialidase assay performed on washed human platelets treated with or without $10 \mu \mathrm{M}$ Ticagrelor and exposed to WT SA for $1 \mathrm{~h}$. Where applicable, all data represented as mean \pm SEM and are representative of at least 3 independent experiments. Statistical significance determined by one way ANOVA with Bonferroni's multiple comparisons test (A, C, D, E). $* \mathrm{P}<0.05$. PBS, phosphate buffered saline; ns, not significant. ND, not detectable. 


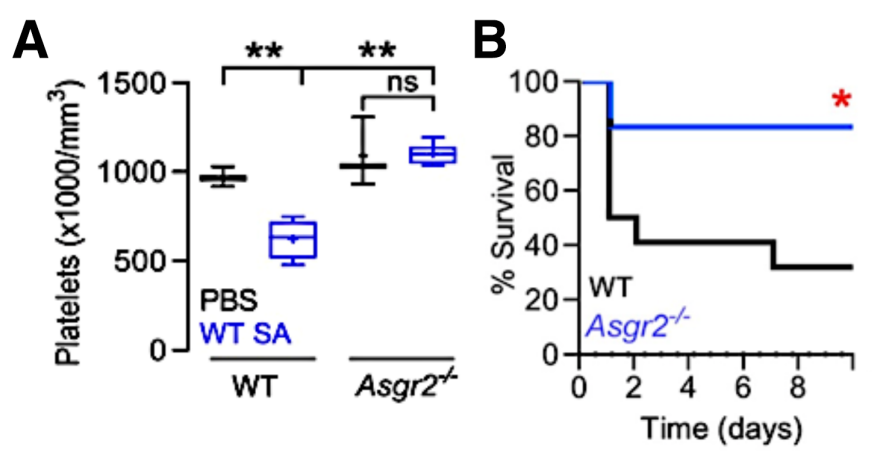

E $\quad$ - Fetuin

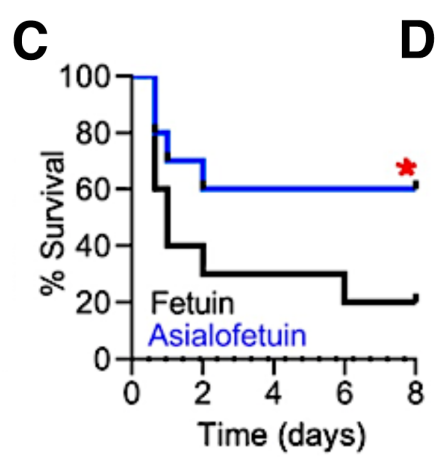

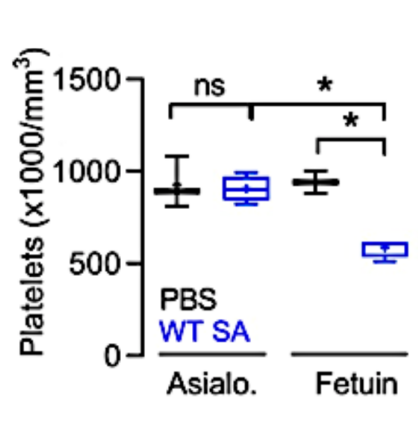

$\mathbf{F}$
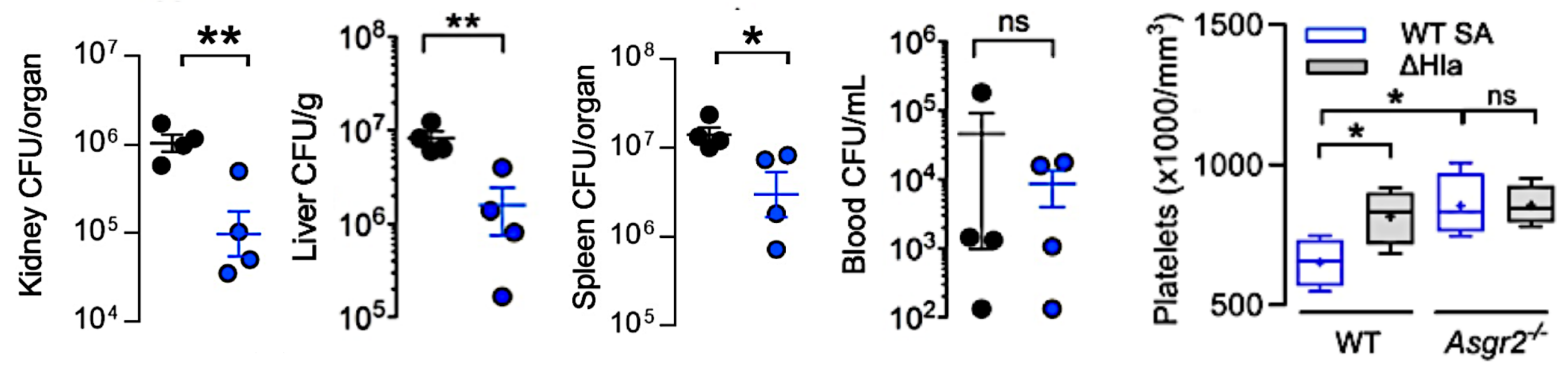

Fig. 5. Inhibition of the hepatic Ashwell-Morell receptor (AMR) supports platelet-mediated defense against SA bacteremia. (A) C57/B16 $(n=4)$ and $\operatorname{Asgr}^{-/-}(n=6)$ mice challenged by intraperitoneal injection with SA, blood harvested by cardiac puncture, and platelet count enumerated. (B) 10-day mortality study with C57/B16 $(\mathrm{n}=22)$ and Asgr $2^{-/-}$mice $(n=16)$ challenged by intraperitoneal injection with SA. Study performed two independent times and data pooled. (C) 8-day mortality study with C57/B16 treated with fetuin $(\mathrm{n}=10)$ or asialofetuin $(\mathrm{n}=10)$ and challenged by intraperitoneal injection with SA. (D) C57/B16 mice treated with asialofetuin $(n=4)$ or fetuin $(n=4)$ and challenged by intraperitoneal injection with SA, platelet count enumerated, and (E) kidneys, liver, spleen, and blood harvested $24 \mathrm{~h}$ post infection for bacterial colony forming unit enumeration. (F) C57/B16 and Asgr2/- mice challenged with wild-type MRSA or the isogenic $\Delta$ Hla mutant. $4 \mathrm{~h}$ post-infection, blood harvested by cardiac puncture for enumeration of platelet count. Statistical significance was determined by unpaired two-tailed Student's T-test (E), two-way ANOVA with Bonferroni's multiple comparisons posttest (A,D,F) and Log-rank (Mantel-Cox) Test $(\mathrm{B}, \mathrm{C})$ for the survival curves. For floating bar graphs, + denotes the mean, whiskers represent min. to max, and floating box represents 25 th to 75 th percentile. ${ }^{*} \mathrm{P}<0.05,{ }^{*} \mathrm{P}<0.005$. PBS, phosphate buffered saline; ns, not significant. 

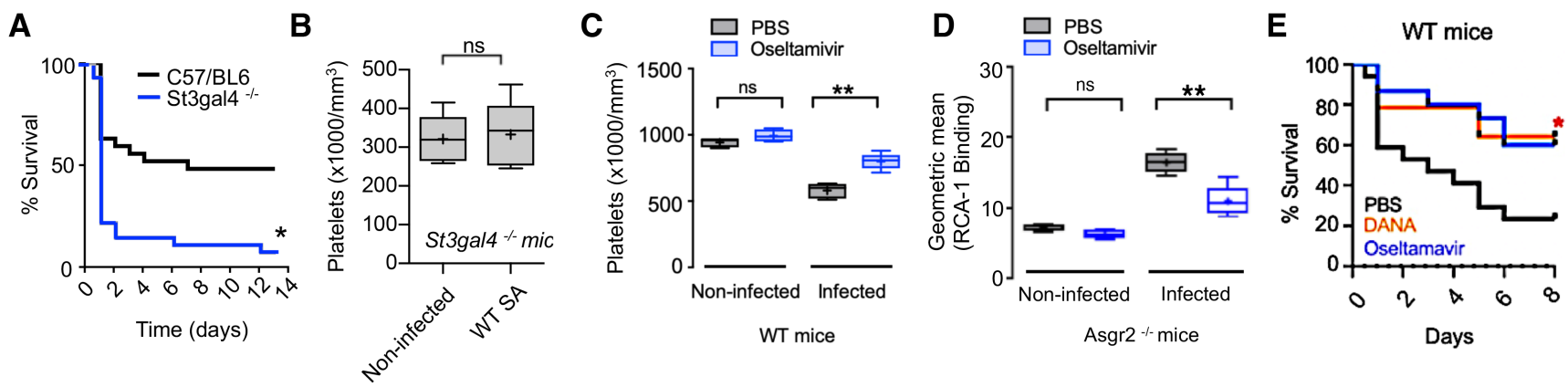

Fig. 6. FDA-approved sialidase inhibitor oseltamivir blocks AMR-mediated platelet clearance and protects against SA bacteremia. (A) St3gal4 ${ }^{-/-}$mice which have decreased platelet sialylation and thrombocytopenia show accelerated mortality upon SA bloodstream infection ( $\mathrm{n}=10$ per group). (B) No difference in circulating platelet count $4 \mathrm{~h}$ after IV SA challenge in WT vs. St3gal4 ${ }^{-/-}$mice $(n=10$ per group $)$. (C) Platelets isolated from Asgr $2^{-/-}$mice treated with or without oseltamavir and infected with MRSA were assessed for RCA-1 lectin binding. (D) C57/B16 mice treated with oseltamavir $(n=6)$ or PBS control $(n=5)$ and infected with WT SA by intraperitoneal injection. Blood harvested $24 \mathrm{~h}$ after infection and platelet counts collected. (DE) 8-day mortality study conducted on C57/B16 mice treated with DANA $(\mathrm{n}=16)$, oseltamavir $(\mathrm{n}=16)$, or PBS control $(\mathrm{n}=16)$. Statistical significance was determined by unpaired two-tailed Student's T-test (B), two-way ANOVA with Bonferroni's multiple comparisons posttest (C,D) and Log-rank (Mantel-Cox) Test (A,E). Where applicable, statistical significance determined by unpaired Two-tailed Student's T-test. For floating bar graphs, + denotes the mean, whiskers represent min. to max, and floating box represents 25 th to 75 th percentile. Unless otherwise stated, ${ }^{*} \mathrm{P}<0.05,{ }^{*} * \mathrm{P}<0.005$. PBS, phosphate buffered saline; ns, not significant. 


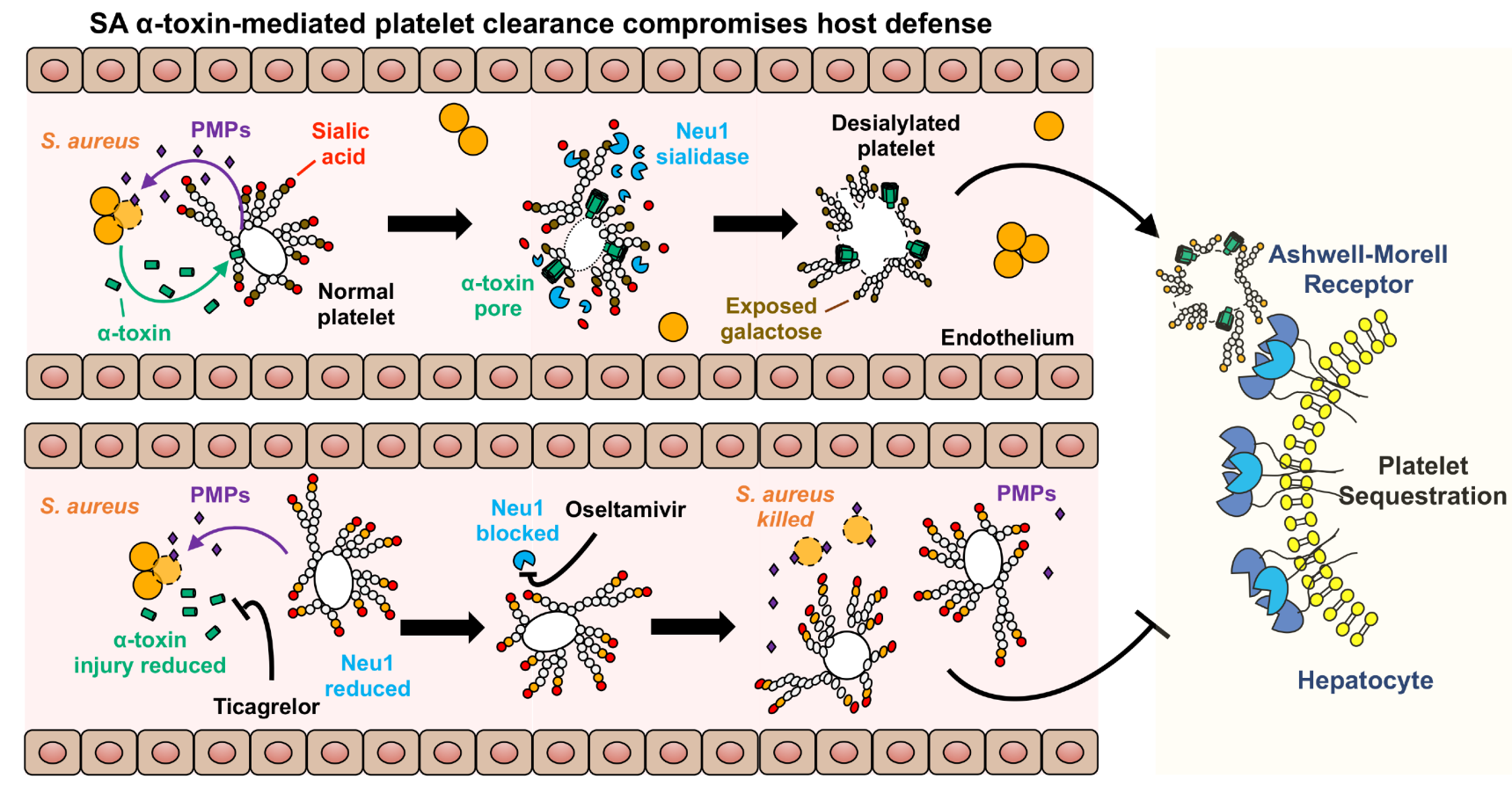

Ticagrelor and oseltamivir preserve platelet counts and antibacterial function

Fig. 7. Schematic illustration of proposed "toxin-platelet-AMR" pathway exploited by SA in the pathogenesis of bloodstream infection. Our experiments suggest the possibility of therapeutic drug repurposing of $\mathrm{P}_{2} \mathrm{Y}_{12}$ (e.g. ticagrelor) and sialidase inhibitor (e.g. oseltamivir) drugs to maintain platelet homeostasis and enhance innate immune clearance of the pathogen. 


\section{Supplementary Materials for}

\section{Repurposed Drugs Block Toxin-Driven Platelet Clearance by the Hepatic Ashwell- Morell Receptor to Clear Staphylococcus aureus Bacteremia}

Josh Sun, Satoshi Uchiyama, Joshua Olson, Yosuke Morodomi, Ingrid Cornax, Nao Ando, Yohei Kohno, May Kyaw, Bernice Aguilar, Nina M. Haste, Sachiko Kanaji, Taisuke Kanaji, Warren E. Rose, George Sakoulas, Jamey D. Marth, Victor Nizet*

*Corresponding author. Email: vnizet@ucsd.edu (V.N.)

The PDF file includes:

Materials and Methods

Fig. S1. Thrombocytopenia established by anti-CD41 antibody treatment of mice.

Fig. S2. Grouping of $\alpha$-toxin expression of SA isolates from bacteremia patients.

Fig. S3. Generation of an isogenic SA $\Delta$ Hla mutant.

Fig. S4. Exclusion of ticagrelor off-target effects on SA immune cell interactions and growth.

Fig. S5. Additional effects of SA or TICA on human platelet phenotypes in vitro.

Fig. S6. Effects of SA challenge on platelets and thrombopoiesis in vivo

Fig. S7. Organ pathology in murine SA infection with or without ticagrelor treatment.

Fig. S8. Neu1 is predominantly detected on the platelet cell surface and is induced by SA exposure

Fig. S9. SA $\alpha$-toxin deletion mutant phenocopies protective effects of TICA, oseltamivir and AMR loss/inhibition.

References (63-69) 


\section{MATERIALS AND METHODS}

\section{Ethics statement}

Animal studies were conducted in accord with protocols approved by the UC San Diego Institutional Animal Care and Use Committee; all efforts were made to minimize animal numbers and suffering. Blood for platelet isolation was obtained via venipuncture from healthy volunteers under written informed consent approved by the UC San Diego Human Research Protection Program.

\section{S. aureus patient isolates}

Consecutive patients from previously published, IRB-approved study (63) and its ongoing continuation (IRB \#2018-0098) with blood cultures of S. aureus (SA) from April 2009 through March 2010 at the University of Wisconsin Hospital (a 493-bed academic medical center in Madison, WI) were analyzed for $\alpha$-toxin expression by western immunoblot and densitometry band analysis by Image J. Levels of $\alpha$-toxin expression was grouped in the following order: low: >10,000; medium: 10,000 - 20,000; high: $>20,000$. Patient demographics, blood work (including platelet and leukocyte counts), and infection source were collected at time of administration. Bacterial isolates obtained at the onset of presentation and stored at $80^{\circ} \mathrm{C}$ until analysis. All laboratory tests were performed by investigators blinded to patient information.

\section{Measurement of desialylation of patient platelets}

In the above patient biobank, $2-5 \mathrm{~mL}$ of patient whole blood is collected on the day of presentation with bacteremia at the hospital. In this analysis, platelets from patients with SA bacteremia were compared to patients with Escherichia coli bacteremia and sepsis, and uninfected healthy volunteers. All patients with S. aureus were diagnosed with bacteremia from an endovascular source by an infectious diseases physician. Patients with E. coli bacteremia/sepsis had infection sources from the urinary or gastrointestinal tract. No patients received P2Y12 antagonists or any other anti-platelet therapy at the time of collection. Samples 
were processed to separate plasma and cells, which are then aliquoted separately stored at $-80^{\circ} \mathrm{C}$ until analysis. Platelets in plasma from patients admitted with SA, Escherichia coli sepsis, or normal controls were analyzed for RCA-1 binding by flow cytometry. Plasma samples were stained with PerCP/Cy5.5 antihuman CD41 antibody (Biolegend) and Fluorescein labeled Ricinus Communis Agglutinin I (RCA-1 : Vector Laboratories). \% desialylated platelets were calculated using the Flojo software.

\section{Bacterial strains and plasmids}

Community-acquired methicillin-resistant SA (MRSA) strain USA300 (TCH1516) and its isogenic $\Delta H$ Ha mutant lacking $\alpha$-toxin, were used in the study. Targeted mutagenesis of Hla was conducted by precise, markerless allelic replacement of USA300 TCH1516 hla gene (Locus tag USA300HOU_1099, NC_010079.1 (1170314.1171273, complement) by PCR-based methods adapting the pKOR1 knock-out strategy previously described for SA mutagenesis (64). As shown in fig. S3, sequence immediately upstream of hla was amplified with the primers D and E and that immediately downstream of $h l a$ with the primers B and F. Primers B and D were constructed with $25 \mathrm{bp} 5$, overhangs for the opposite flanking region. Upstream and downstream PCR products were fused using primers $\mathrm{E}$ and $\mathrm{F}$ in a second round of PCR. The amplicons were then subcloned into temperature-sensitive vector $\mathrm{pKOR} 1$ using the BP clonase reaction (Invitrogen). The resulting plasmid pKOR1-hla was passed through SA RN4220, and the purified plasmid electroporated into strain TCH1516. Precise in-frame allelic replacement of hla was established by a two-step process of temperature shifting and anti-sense counterselection and confirmed by PCR. Other listed primers (fig. S3) were used for PCR confirmations and for screening potential mutants and clones. All strains were routinely grown in Todd Hewitt broth (THB) and propagated shaking at $37^{\circ} \mathrm{C}$ to mid-log phase (optical density $\left.600 \mathrm{~nm}\left(\mathrm{OD}_{600}\right)=0.4\right)$ unless otherwise stated. Bacteria were collected by centrifugation at 4,000 RPM x 10 min, washed once in phosphate-buffered saline (PBS), and resuspended to the desired dilution in PBS. 


\section{Platelet and neutrophil isolation}

For platelet isolation, human venous blood was drawn using a $20 \mathrm{G}$ needle from healthy human donors using acid-citrate-dextrose buffer (ACD; Sigma) as an anticoagulant (1:6 v/v), unless otherwise stated. To obtain platelet-rich plasma (PRP), blood was centrifuged at 1,000 RPM x 10 min with no brake. To avoid contaminations with other cell types, only the upper two thirds of the platelet-rich plasma fractions were used. PRP was centrifuged at 1.500 RPM x $10 \mathrm{~min}$. Isolated platelets were resuspended in serum-free, antibiotic-free, inhibitor-free RPMI (without phenol red) at room temperature. Blood was drawn according to a protocol approved by the local ethics committee. For neutrophil isolation, venous blood was drawn from healthy donors as above but using heparin as an anticoagulant. Purified neutrophils were collected using Polymorph Prep (Axis-Shield, Dundee, Scotland) per manufacturer's protocol.

\section{Platelet cytotoxicity}

Human platelets were pre-treated with $10 \mu \mathrm{M}$ ticagrelor (Sigma) or vehicle control and incubated with rotation at $37^{\circ} \mathrm{C}+5 \% \mathrm{CO}_{2}$ for $20 \mathrm{~min}$, then exposed to $5 \mu \mathrm{g} / \mathrm{mL}$ recombinant $\alpha$-toxin (H9395 Sigma) for an additional $30 \mathrm{~min}$. Samples were spun down at $500 \mathrm{x} \mathrm{g}$ for $5 \mathrm{~min}$, and supernatants evaluated using a commercial lactate dehydrogenase (LDH) assay (Promega) or ATP assay (Promega, CellTiter-Glo® Luminescent Cell Viability Assay).

\section{Human platelet sialidase (neuraminidase) assay}

Washed human platelets $\left(3 \times 10^{7}\right)$ were assayed for sialidase activity using a slight modification of the previously published 2'-(4-methylumbelliferyl)- $\alpha-\mathrm{D}-N$-acetylneuraminic acid (4MU; Sigma) assay (65, 66). Platelets were added to a white 96 -well plate (Costar), pretreated with or without the identified drugs for $15 \mathrm{~min}$, and exposed to $3 \times 10^{7} \mathrm{CFU}$ of $\mathrm{SA}(\mathrm{MOI}=1: 1)$ for $1 \mathrm{~h}$ at $37^{\circ} \mathrm{C}$ in $5 \% \mathrm{CO}_{2}$. Next, $125 \mu \mathrm{M} 4 \mathrm{MU}$ was added and incubated $30 \mathrm{~min}$ at $37^{\circ} \mathrm{C}$ before $1 \mathrm{M} \mathrm{Na}_{2} \mathrm{CO}_{3}$ was added to each sample and fluorescence 
determined at excitation $530 \mathrm{~nm}$ and emission $585 \mathrm{~nm}$. Background fluorescence was measured following the same procedure but in the presence of $1 \mathrm{mM}$ sialidase inhibitor DANA.

\section{Human platelet ADAM10 protease assay}

Human platelets were isolated from healthy donors using hirudin as an anticoagulant. Isolated platelets were resuspended in serum-free, antibiotic-free, inhibitor-free RPMI (without phenol red) at room temperature. Human platelets $\left(2 \times 10^{7}\right)$ were pre-treated with $10 \mu \mathrm{M}$ ticagrelor (Sigma) or vehicle control and incubated at $37^{\circ} \mathrm{C}$ with rotation for $20 \mathrm{~min}$, at which time platelets were exposed to $5 \mathrm{mg} / \mathrm{mL}$ recombinant $\alpha$-toxin and a fluorogenic ADAM10 specific substrate (PEPMCA001, Biozyme) at $37^{\circ} \mathrm{C}+5 \% \mathrm{CO} 2$ without shaking. Fluorescence was measured every $15 \mathrm{~min}$ for $2.5 \mathrm{~h}$ using wavelengths of $325 \mathrm{~nm}$ (excitation) and $393 \mathrm{~nm}$ (emission).

\section{Calcium assay (Fluo-3 assay)}

Human donor blood was collected using hirudin as an anticoagulant. Platelet-rich plasma (PRP) was isolated and incubated with $2 \mathrm{mM}$ Fluo-3 AM (Thermo Fisher Scientific) at $37^{\circ} \mathrm{C}+5 \% \mathrm{CO} 2$ with rotation for $20 \mathrm{~min}$. Isolated platelets were resuspended in serum-free, antibiotic-free, inhibitor-free RPMI (without phenol red) at room temperature, pre-treated with $10 \mu \mathrm{M}$ Ticagrelor (Sigma) or vehicle control for $15 \mathrm{~min}$, then exposed to $5 \mu \mathrm{g} / \mathrm{mL}$ recombinant $\alpha$-toxin. Fluorescence was measured at $505 \mathrm{~nm}$ excitation and 530 nm emission every $30 \mathrm{sec}$.

\section{Transmission electron microscopy}

Isolated human platelets were pre-treated with $10 \mu \mathrm{M}$ ticagrelor (Sigma) or vehicle control at $37^{\circ} \mathrm{C}+5 \%$ $\mathrm{CO}_{2}$ for $20 \mathrm{~min}$, then infected with MRSA at $\mathrm{MOI}=0.01$ for an additional $2 \mathrm{~h}$. Samples were fixed in modified Karnovsky's fixative $(2.5 \%$ glutaraldehyde $+2 \%$ paraformaldehyde in $0.15 \mathrm{M}$ sodium cacodylate buffer, $\mathrm{pH}$ 7.4) for at least $4 \mathrm{~h}$, post-fixed in 1\% osmium tetroxide in $0.15 \mathrm{M}$ cacodylate buffer for $1 \mathrm{~h}$, and 
stained in block in 2\% uranyl acetate for $1 \mathrm{~h}$. Samples were dehydrated in ethanol, embedded in Durcupan epoxy resin (Sigma-Aldrich), sectioned at 50-60 nm on a Leica UCT ultramicrotome, and picked up on Formvar and carbon-coated copper grids. Sections were stained with $2 \%$ uranyl acetate for 5 min and Sato's lead stain for $1 \mathrm{~min}$. Grids were viewed using a Tecnai G2 Spirit BioTWIN transmission electron microscope and photographs were taken with an Eagle 4k HS digital camera (FEI). Images were taken from multiple random fields at $1200 \times, 2900 \times, 23,000 \times$; gross morphology was analyzed in a blinded fashion.

\section{Bacterial growth curves}

Sterile non-pyrogenic tubes containing THB treated with ticagrelor $(10 \mu \mathrm{M})$ or untreated were inoculated with overnight bacterial cultures to achieve an optical density $(600 \mathrm{~nm})$ of 0.1 . Tubes were incubated in a shaking $37^{\circ} \mathrm{C}$ incubator, and absorbance measured every $30 \mathrm{~min}(600 \mathrm{~nm})$ for $8 \mathrm{~h}$ using a Spectronic 20D+ spectrophotometer (Thermo Scientific, Waltham, MA, USA)

\section{Bactericidal assays}

Human platelet killing: Isolated platelets were pre-treated with $10 \mu \mathrm{M}$ ticagrelor (Sigma) or vehicle control for $20 \mathrm{~min}$ at $37^{\circ} \mathrm{C}+5 \% \mathrm{CO}$ for $20 \mathrm{~min}$, then infected with MRSA at MOI $=0.01$ for $2 \mathrm{~h}$. Infected platelets were sonicated (Fisher Sonic Dismembrator 550) for $3 \mathrm{sec}$, serially diluted, and plated THA plates. Percent killing by platelets as surviving CFU vs. original inoculum. Human neutrophil killing: Freshly isolated human neutrophils (PolymorphPrep) in serum-free RPMI were added to 96-well plates at $5 \times 10^{4}$ cells per well and treated with ticagrelor $(10 \mu \mathrm{M})$ or vehicle control for $1 \mathrm{~h}$ at $37^{\circ} \mathrm{C}+5 \% \mathrm{CO}_{2}$, were infected with MRSA at MOI $=0.01$ for $2 \mathrm{~h}$. After incubation, cells were lysed with $0.025 \%$ Triton $\mathrm{X}-100$, serial diluted, and plated on THA for CFU enumeration and determination of percent killing vs. inoculum. THP-1 macrophage killing: The THP-1 monocyte cell line authenticated by ATCC were cultured in RPMI (with phenol red) medium $+10 \%$ fetal bovine serum (FBS). Cells were differentiated in a 96 well format for 48 $\mathrm{h}$ with $25 \mathrm{nM}$ phorbol myristate acetate (PMA, Sigma) with a subsequent $24 \mathrm{~h}$ rest period in RPMI $+10 \%$ 
FBS. On the day of infection, cells were washed once with PBS, treated with ticagrelor $(10 \mu \mathrm{M})$ or untreated control for $1 \mathrm{~h}$, and infected with MRSA at MOI 1:100. After incubation, cells were lysed with $0.025 \%$ triton X-100, serial diluted, and plated on THA for CFU enumeration and determination of percent killing vs. inoculum. Human whole blood killing: Whole blood was drawn from healthy donors using anticoagulant citrate dextrose solution (ACD, 1:6). Blood was pre-treated with varying concentrations of ticagrelor or vehicle control at $37^{\circ} \mathrm{C}+5 \% \mathrm{CO}_{2}$ with rotation, then infected with MRSA (1:10) and incubated for an additional $1 \mathrm{~h}$. After incubation, samples were sonicated (Fisher Sonic Dismembrator 550) twice at 20\% maximum power for $3 \mathrm{sec}$ with 10 seconds interval, serially diluted, and plated on THA for CFU enumeration and determination of percent killing vs. inoculum.

\section{Induction and quantification of neutrophil extracellular traps}

To induce extracellular trap production, neutrophils were seeded in 96-well plates at a density of $5 \times 10^{4}$ cells per well in RPMI (without phenol red). Cells were incubated with ticagrelor (10 uM) or untreated control at $37^{\circ} \mathrm{C}$ with $5 \% \mathrm{CO}_{2}$ for $1 \mathrm{~h}$ before addition of NET-inducing PMA $(25 \mathrm{nM})$ for an additional. Extracellular DNA content was quantified using a Quant-IT PicoGreen dsDNA Assay Iit (Life Technologies, Carlsbad, CA) per manufacturer's instructions.

\section{Human platelet surface P-selectin, ADAM-10, GP6 and CD63 measurements}

The surface expression of P-selectin, ADAM-10, GP6 and CD63 were measured by flow cytometry. Human platelets were first incubated with TICA or vehicle control for $20 \mathrm{~min}$ in $37^{\circ} \mathrm{C}$. After a wash with PBS, $1 \times 10^{7}$ platelets were incubated with SA, $\alpha$-toxin or control media for $90 \mathrm{~min}$. In each tube, antibodies against P-selectin, ADAM-10, GP6 or CD63 each conjugated with PE (Biolegend, San Diego) were added. Expression of each molecule on the platelet surface was detected using FACSCalibur (BD) and analyzed using Flo Jo software (Flojo LLC). 


\section{Human platelet $\beta$-galactosidase activity}

Human platelets were incubated with RPMI only, SA or SA and ticagrelor. After 90 min incubation at $37^{\circ} \mathrm{C}$, the samples were centrifuged at $2000 \mathrm{rpm}$ for $10 \mathrm{~min}$ to collect supernatants. $\beta$-galactosidase activity in platelet supernatants was measured using the Mammalian $\beta$-Galactosidase Assay (Thermo-Fisher) per the manufacturer's protocol.

\section{Mouse infection and platelet count determination}

Wild-type SA and isogenic $\Delta \mathrm{Hla}$ mutant cultures were grown shaking overnight at $37^{\circ} \mathrm{C}$ in $\mathrm{THB}$, washed once in $1 \times$ PBS, and $1 \times 10^{8}$ colony forming units (CFU) were injected intravenously (i.v.) into outbred 8to 10-week-old CD1 mice (Charles Rivers Laboratories). Blood was collected, platelet count determined, and samples serial diluted and plated onto Todd Hewitt agar (THA) plates for CFU enumeration. Platelet count and CFU burden were enumerated $4 \mathrm{~h}$ post infection. For platelet depletion studies, endotoxin and azide-free $1 \mathrm{mg} / \mathrm{kg}$ anti-CD41 antibody (clone MWReg30, Biolegend) or $1 \mathrm{mg} / \mathrm{kg}$ isotype control rat IgG1 (clone RTK2071, Biolegend) were injected intraperitoneally (i.p.). 1 x $10^{8}$ SA was administered by tail vein injection (i.v.) $16 \mathrm{~h}$ post-antibody treatment, and $4 \mathrm{~h}$ later mice were euthanized by $\mathrm{CO}_{2}$ inhalation, blood collected by cardiac puncture with a $25 \mathrm{G}$ needle attached to a syringe containing $100 \mathrm{~mL}$ ACD buffer, and a complete blood count (CBC) was obtained. Blood, liver, spleen and kidneys were harvested, homogenized, and plated in serial dilutions onto THA plates for CFU counts. For ex vivo platelet depletion analysis, mice were treated as above and blood collected by cardiac puncture $16 \mathrm{~h}$ after antibody injection, and $1 \times 10^{6} \mathrm{SA}$ added to the platelet-depleted and control blood and incubated at $37^{\circ} \mathrm{C}$ with rotation for $1 \mathrm{~h}$ prior to dilution plating for CFU counts. For platelet count determination in a time course after infection, 5 x $10^{7}$ SA were administered i.v. an $50 \mu$ l blood collected by submandibular bleeding into EDTA tubes at each time point. Platelet counts were analysed using ProCyte Dx Hematology Analyzer (IDEXX). 


\section{Measurement of GP6 expression on murine platelets}

GP6 expression on murine platelets during SA systemic infection was measured by flow cytometry. Blood collected above for time course platelet count was incubated with anti-mouse GP6 antibody (Emfret). Expression of GP6 and percentage GP6 negative platelets were analyzed using Flo Jo software (Flojo LLC).

\section{Analysis of bone marrow megakaryocyte ploidy in murine SA systemic infection}

Measurement of megakaryocyte counts in bone marrow and evaluation of megakaryocyte ploidy was performed as described previously (67). In short, BM cells were harvested from femurs of mice $72 \mathrm{~h}$ after SA $\left(5 \times 10^{7} \mathrm{CFU}\right)$ i.v. infection $(\mathrm{n}=3$ in each group). After gently suspending the BM cells in $0.5 \%$ BSA PBS with $2 \mathrm{mM}$ EDTA, cells were fixed in $2 \%$ paraformaldehyde at $4{ }^{\circ} \mathrm{C}$ for $2 \mathrm{~h}$, then washed and resuspended in PBS/EDTA with Fc Block for $10 \mathrm{~min}$. Next $1 \mu \mathrm{g} / \mathrm{mL}$ Brilliant Violet $421^{\mathrm{TM}}$ anti-mouse CD41 antibody (Biolegend,), $75 \mu \mathrm{g} / \mathrm{mL}$ propidium iodide (Sigma-Aldrich) and $45 \mu \mathrm{g} / \mathrm{mL}$ RNAse A (Qiagen) were added and incubated for $30 \mathrm{~min}$. Equal volumes of each sample (100 $\mu 1)$ were analyzed by flow cytometry using a NovoCyte (ACEA Biosciences, San Diego, CA). Megakaryocytes were identified as CD41+ cells and megakaryocyte number/ploidy was analyzed for 4 min.

\section{Platelet microparticle analysis by flow cytometry}

Isolated human platelets were infected with SA strains for various time points. After each time point, samples were collected, centrifuged and the supernatant analyzed using flow cytometry for microparticle content by adding FITC-conjugated anti-CD41 antibody (Biolegend). FITC positive particles smaller than $1 \mu \mathrm{m}$ latex beads were selected. The absolute count was calculated from the $15 \mu \mathrm{m}$ beads added to the same samples. For counting platelet microparticles in mouse serum, animals were infected with 1 x $10^{8}$ SA i.v., blood collected $4 \mathrm{~h}$ after infection, and the serum separated. After adding FITC conjugated anti-CD41 antibody, microparticles in serum were analyzed by FACS as described above. 


\section{Asgr 2 ${ }^{-/-}$, St3gal4---and AMR inhibitor mouse infection studies}

Eight- to 12-week-old $\mathrm{Asgr}^{-/-}$mice (68) or 10 to 14-week-old St3gal4 ${ }^{-/}$mice on a C57/B16 (Jackson Laboratories) genetic background (69) and WT mice bred and raised in the same room were used. WT SA was grown overnight shaking at $37^{\circ} \mathrm{C}$ in THB, washed once in $1 \times$ PBS, and $1 \times 10^{8}$ colony forming units (CFU) injected intraperitoneally (i.p.) unless otherwise specified in the Figure Legend, and mortality observed over the course of 10 days. For AMR inhibitor studies, C57/B16 mice were treated with $25 \mathrm{mg} / \mathrm{mL}$ asialofetuin or fetuin prior to i.p. challenge with $1 \times 10^{8} \mathrm{CFU} \mathrm{SA}$; mortality was observed over the course of 8 days. For both studies, mice that appeared moribund were euthanized by $\mathrm{CO}_{2}$ asphyxiation. Platelet count enumerations were performed $4 \mathrm{~h}$ post infection. For AMR inhibitor CFU enumeration, mice were euthanized $24 \mathrm{~h}$ post-infection, organs harvested, and dilution plated onto THA.

\section{Sialidase inhibitor mouse infection studies}

Eight- to 10-week-old wild-type C57/B16 mice were treated with oseltamivir (5 mg/kg) in $100 \mu \mathrm{L}$ PBS or Neu1-selective inhibitor C9-butyl-amide-2-deoxy-2,3-dehydro-N-acetylneuraminic acid (DANA) (2 $\mathrm{mg} / \mathrm{kg}$ ) at the time of- and 3 hours-post intraperitoneal infection with $1 \times 10^{8} \mathrm{CFU}$ SA. Platelets were enumerated and sialidase activity assessed using $2^{\prime}$-(4-methylumbelliferyl)- $\alpha$-D- $N$-acetylneuraminic acid (4MU; Sigma) from blood collected $4 \mathrm{~h}$ post infection per using a previously described protocol (4). For ex vivo sialidase analysis, murine platelet rich plasma (PRP) was isolated by cardiac puncture with a $25 \mathrm{G}$ needle attached to a syringe containing $100 \mu \mathrm{L}$ ACD and centrifuged at $100 \mathrm{x} g$ for $10 \mathrm{~min}$ without braking. Following isolation, $25 \mu \mathrm{L}$ of PRP was added to wells of white 96-well plate (Costar) with $25 \mu \mathrm{L}$ RPM I+ $125 \mu \mathrm{M} 4 \mathrm{MU}$. The plate was incubated at $37^{\circ} \mathrm{C}+5 \% \mathrm{CO}_{2}$ for $30 \mathrm{~min}$, followed by an addition of $1 \mathrm{M}$ $\mathrm{Na}_{2} \mathrm{CO}_{3}$, and fluorescence determined at excitation $530 \mathrm{~nm}$ and emission $585 \mathrm{~nm}$. 


\section{Ticagrelor treatment mouse infection studies}

SA was grown shaking overnight at $37^{\circ} \mathrm{C}$ in THB, washed once in PBS, and $1 \times 10^{8}$ colony forming units (CFU) injected intravenously (i.v.) into outbred 8- to 10-week-old CD1 (Charles River Laboratories, Wilmington, MA, USA) mice. Where indicated, ticagrelor (4 mg/kg) or vehicle (water) was delivered by oral gavage $24 \mathrm{~h}$ prior- and every $24 \mathrm{~h}$ post-infection over a course of 10 days. Mice that appeared moribund were euthanized by $\mathrm{CO}_{2}$ asphyxiation. For quantification of $\mathrm{CFU}$ burden and histological preparation, mice treated with ticagrelor $(4 \mathrm{mg} / \mathrm{kg}$ ) or vehicle (water) $12 \mathrm{~h}$ prior- and every $24 \mathrm{~h}$ post-intravenous injection of $1 \times 10^{8} \mathrm{CFU}$ SA. At $12 \mathrm{~h}$ post-infection, two mice from each group were euthanized by $\mathrm{CO}_{2}$ asphyxiation and the kidneys, spleen, heart, and liver harvested and fixed 10\% neutral-buffered formalin for $24 \mathrm{~h}$, then routinely processed and paraffin-embedded for histological analysis. Five-micron thick hematoxylin and eosin-stained sections of each tissue were examined by a veterinary pathologist blinded to the treatment group. Distinct bacterial colonies visible at $4 \mathrm{X}$ magnification were counted in three longitudinal sections of heart and six longitudinal sections of kidney. Lesions related to bacterial infection were described and graded (minimal-1, mild-2, moderate-3, or severe-4) based on degree of tissue damage. At $72 \mathrm{~h}$ post infection, remaining surviving mice were euthanized by $\mathrm{CO}_{2}$ asphyxiation, blood collected by cardiac puncture, and organs excised. Blood and organ homogenate (MagNA Lyser instrument (Roche Diagnostics Corporation, Indianapolis, IN) were serially diluted in molecular grade $\mathrm{H}_{2} \mathrm{O}$ and plated onto THA for bacterial CFU enumeration. The study was performed three independent times and data from a representative experiment shown. For platelet quantification, mice were treated with ticagrelor $(4 \mathrm{mg} / \mathrm{kg})$ or vehicle (water) every $12 \mathrm{~h}$ for $72 \mathrm{~h}$ prior to i.v. injection of $1 \times 10^{8} \mathrm{CFU}$ SA. Four h post-infection, blood was collected by cardiac puncture with a $25 \mathrm{G}$ needle attached to a syringe containing $100 \mathrm{~mL}$ ACD buffer, transferred into EDTA tubes and a complete blood count $(\mathrm{CBC})$ was obtained. 


\section{Mouse infection studies with the SA $\Delta$ hla mutant}

Mice treated with ticagrelor, oseltamivir, asialofetuin or Asgr2-/- mice were infected with 8 x $10^{8}$ SA $\Delta$ Hla mutant strain i.v. and platelet counts and bacterial burden analyzed as described above.

\section{Statistics}

All in vitro, ex vivo, and in vivo data were collected from three or more $(\geq 3)$ independent experiments with $\geq 3$ biological replicates and are represented as mean \pm standard error mean (SEM), unless otherwise stated. For descriptive data (histopathologic staining), experiments were performed at least twice independently with $\geq 3$ biological replicates and illustrated as best representative images. The alpha level used for all tests was 0.05; unpaired Student's t-test, one-way ANOVA with Bonferroni's multiple comparisons test or twoway ANOVA with Bonferroni's multiple comparisons test was performed as explained in figure legends to determine statistical significance. Statistical analyses were done using GraphPad Prism, version 8.42 (GraphPad Software Inc., La Jolla, CA, USA). P values $<0.05$ were considered statistically significant. 


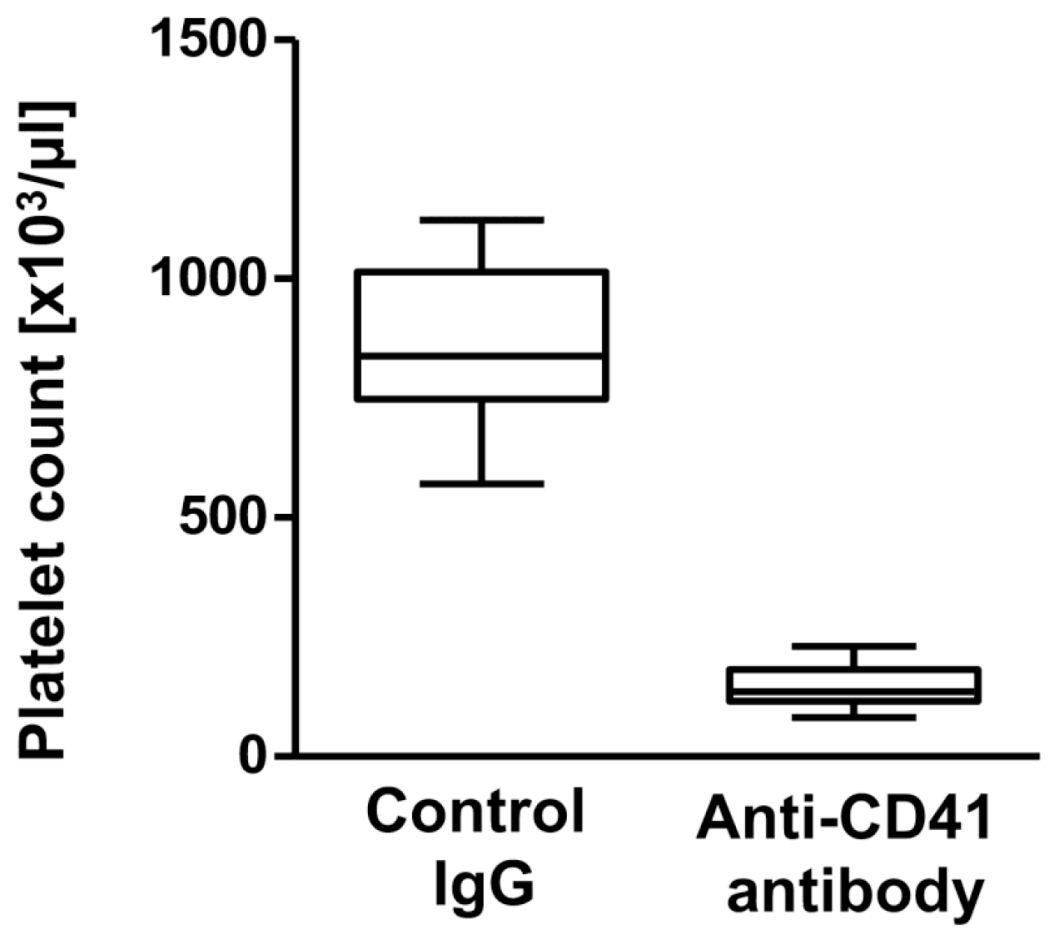

Fig. S1. Thrombocytopenia established by anti-CD41 antibody treatment of mice. Endotoxin and azide-free $1 \mathrm{mg} / \mathrm{kg}$ anti-CD41 antibody (clone MWReg30, Biolegend) or $1 \mathrm{mg} / \mathrm{kg}$ isotype control rat IgG1 (clone RTK2071, Biolegend) were injected intraperitoneally (i.p.) and blood collected by for platelet enumeration $16 \mathrm{~h}$ post-antibody treatment. 


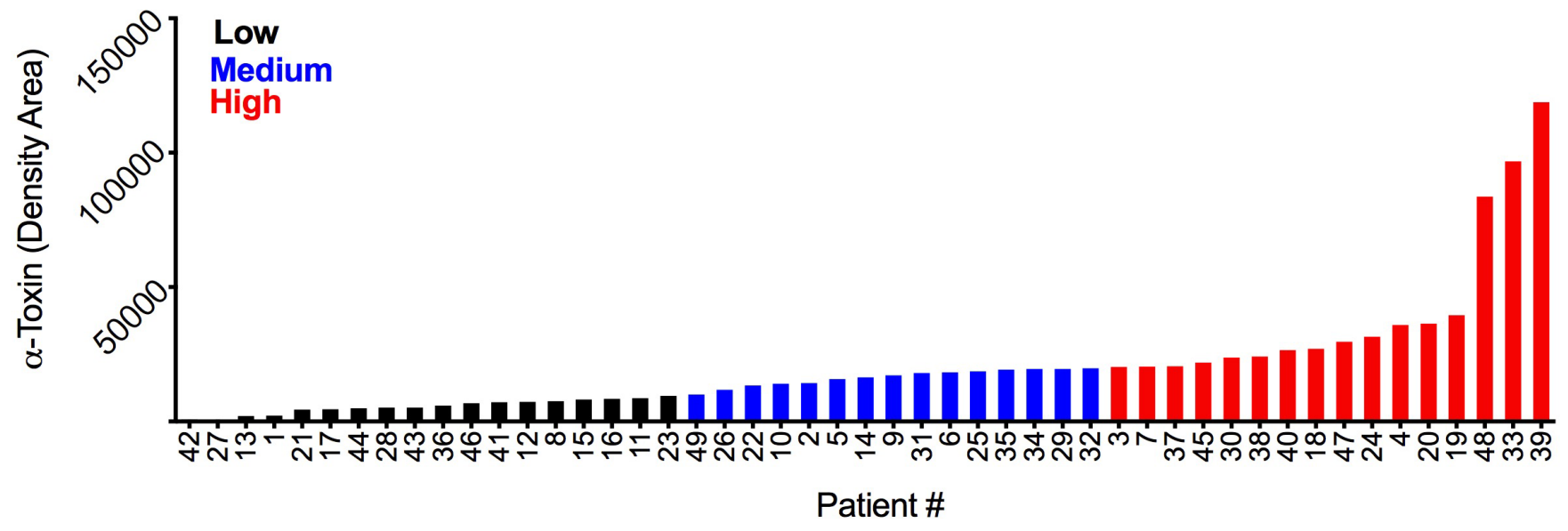

Fig. S2. Grouping of $\boldsymbol{\alpha}$-toxin expression of SA isolates from bacteremia patients. Western immunoblot band analysis on 49 patient clinical isolates grouped according to density area. Groups: low $<10,000$ (black); med. $<20,000$ (blue); high $>20,000$ (red). Densitometry performed using ImageJ software. Data represented as mean only. 

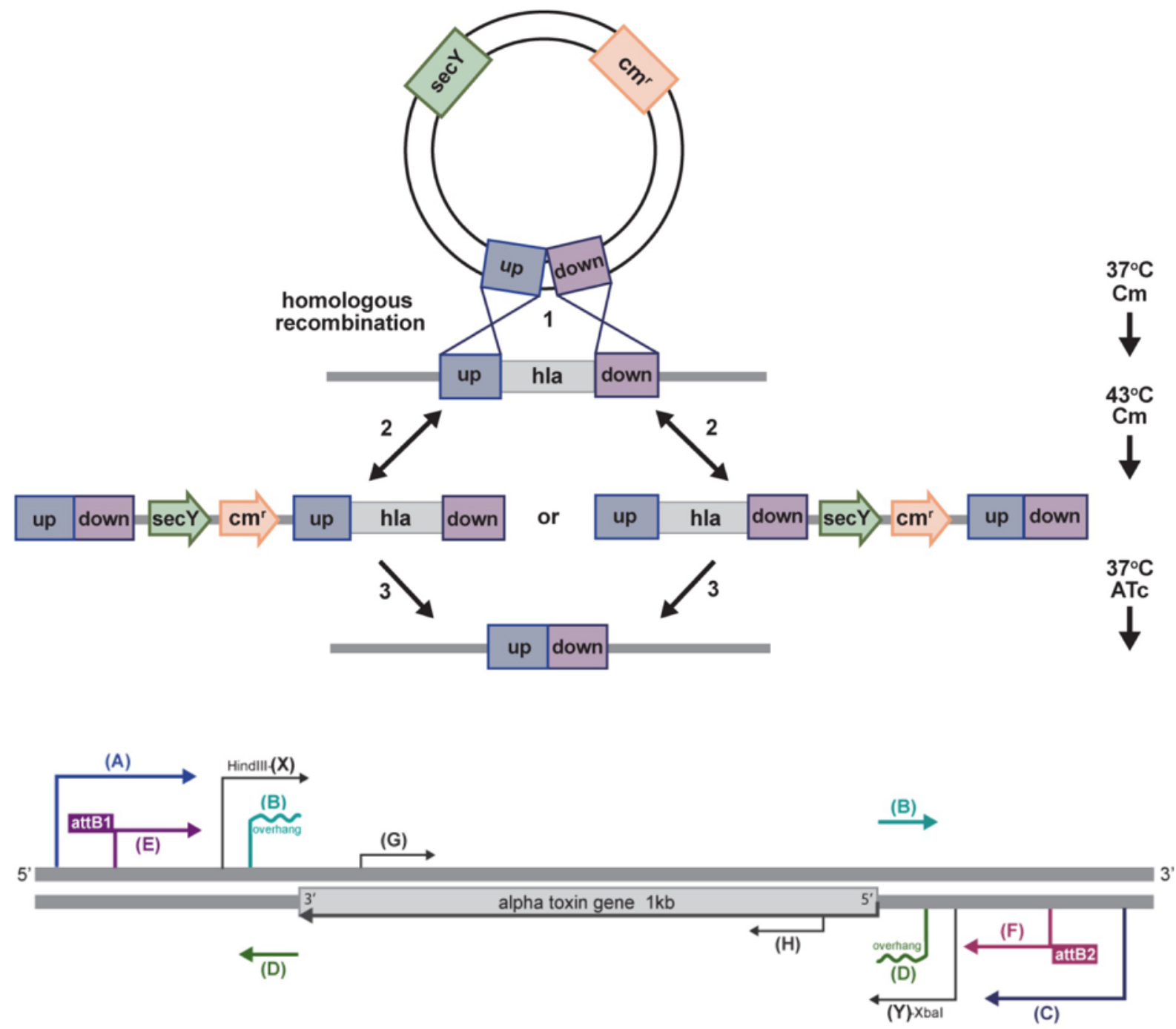

\section{Primers}
A UpConstructUp 5'-gatgaagaagtccatacaaaatccgcatcatttg-3'
B DownConstructUp 5'-catgaacaaataatttacatttcatcatccttctatttttaaaac-3'
C DownConstructDwn 5'-catgggcatttcattcggtcaactactaccaatataatattgtagagcctaag-3'
D UpConstructDown 5'-aatagaaggatgatgaaatgtaaattatttgttcatgtca-3'
E $\underline{\text { attB1 }}+$ Up
5'-GGGGACAAGTITGTACAAAAAAGCAGGCTgaaattaatcatgcatg-3'
F Down $+\underline{\text { attB2 }}$
G Confirm $F$
H Confirm R 5'-GGGGACCACTITGTACAAGAAAGCTGGGTtattccttcatttaatg-3' 5'-ccaatttgttgaagtccagtgcaattgg-3' 5'-cctgtcgtcaatgccgcagattct-3'

Fig. S3. Generation of an isogenic SA $\Delta$ Hla mutant. Scheme and primer selection for PCR-based targeted markerless allelic replacement mutagenesis of the hla gene encoding $\alpha$-toxin in methicillin-resistant SA strain USA300 TCH1516. 
A

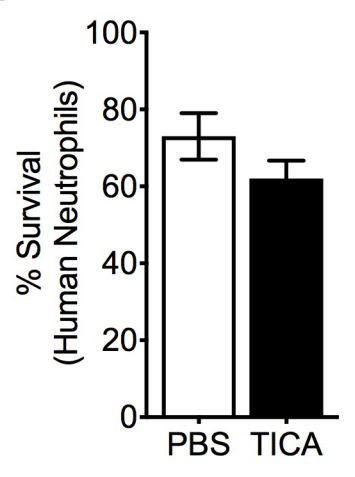

B

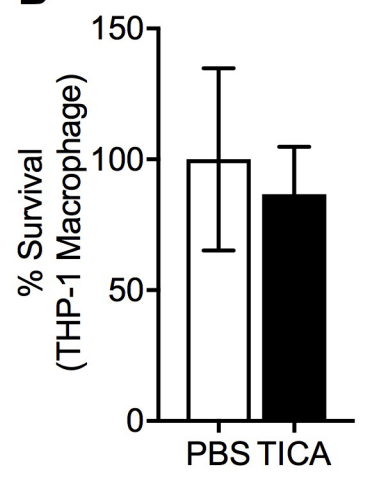

C

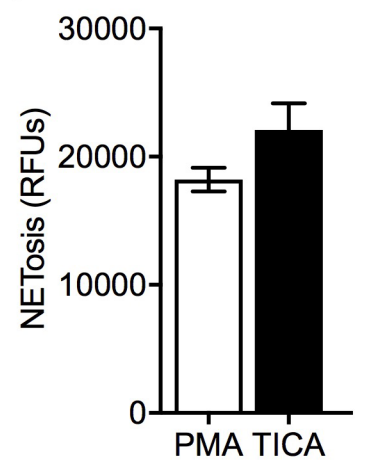

D

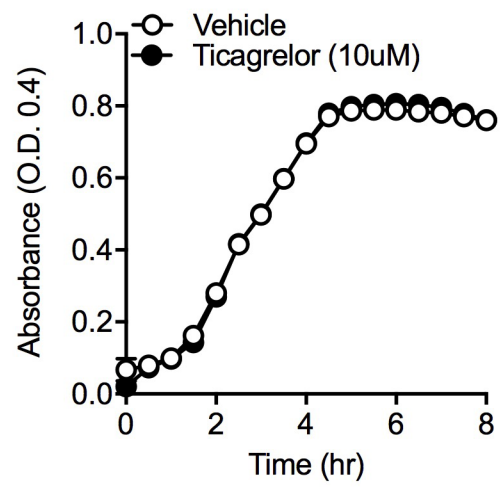

Fig. S4. Exclusion of ticagrelor off-target effects on SA immune cell interactions and growth. (A) Quantification of MRSA colony-forming units (CFUs) in a purified human neutrophil killing assay. Human neutrophils and (B) THP-1-derived macrophages treated with vehicle control (PBS 1x) or $10 \mu \mathrm{M}$ Ticagrelor for 30 minutes prior to $1 \mathrm{~h}$ exposure to MOI: $1 \mathrm{MRSA}$. (C) Isolated human neutrophils pre-treated with vehicle or $10 \mu \mathrm{M}$ Ticagrelor and subsequently exposed to PMA for quantification of neutrophil extracellular trap (NET) production. (D) Growth curve analysis of pre-treated SA with $10 \mu \mathrm{M}$ TICA. Absorbance measured at an optical density (O.D.) of 0.4 measured over the course of 8 hours. Where applicable, data represented as mean \pm SEM and are representative of at least three independent experiments. Statistical significance determined by unpaired Two-tailed Student's $t$ test. $* P<0.05$ 

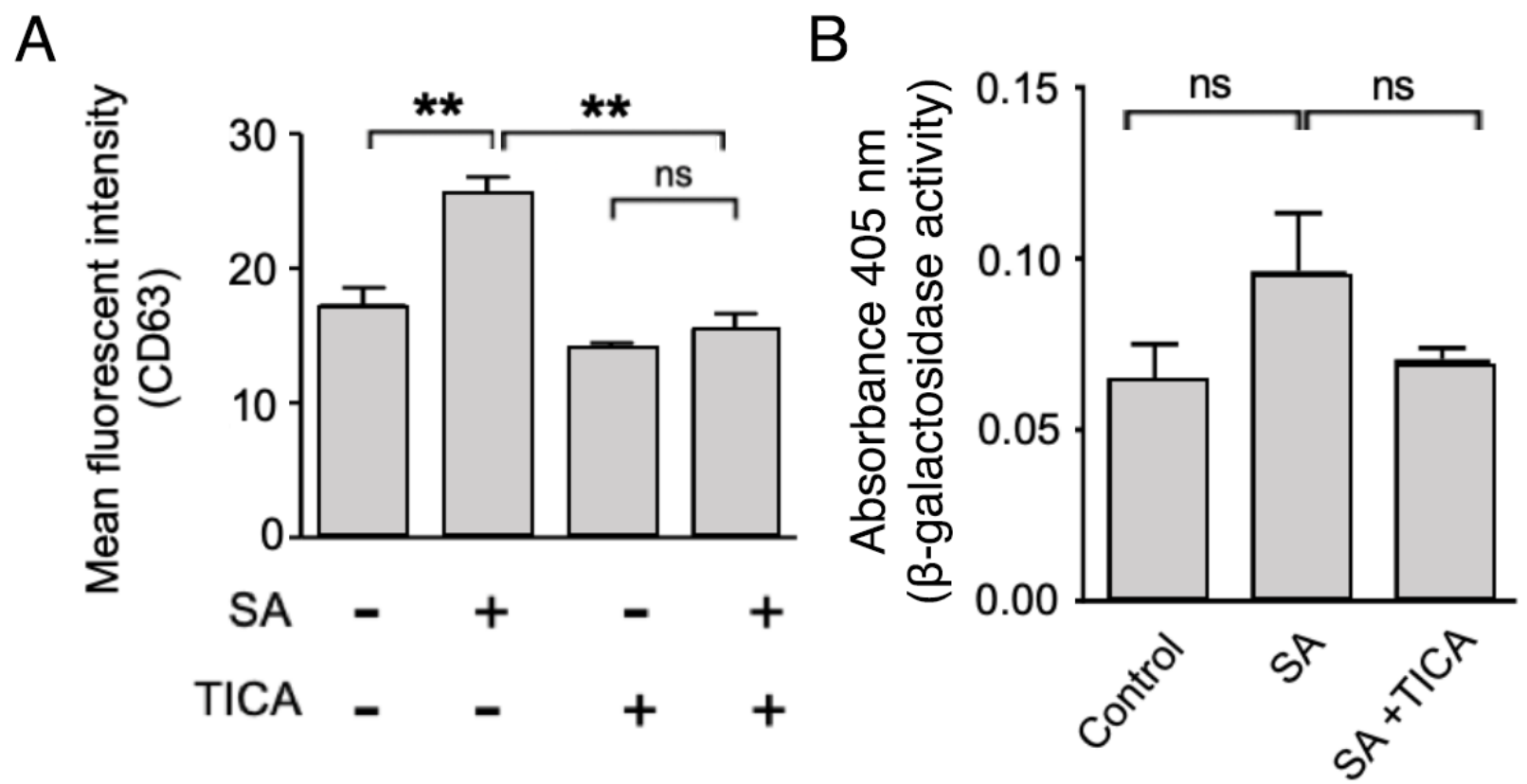

Fig. S5. Additional effects of SA or TICA on human platelet phenotypes in vitro. (A) Human platelet CD63 expression was measured by flow cytometry with or without TICA treatment and MRSA challenge $(\mathrm{MOI}=0.1)$ after $90 \mathrm{~min}$. (B). $\beta$-Galactosidase activity released from human platelets were measured using the colorimetric method of the Mammalian $\beta$-Galactosidase Assay Kit (Thermo-Fisher). All data are represented as mean \pm SEM and are representative of at least 3 independent experiments. Statistical significance was determined by one-way ANOVA with Bonferroni's multiple comparisons test (A,C,G), unpaired two-tailed Student's T-test $(\mathrm{H})$ and two-way analysis of variance (ANOVA) with Bonferroni's multiple comparisons posttest (I). 

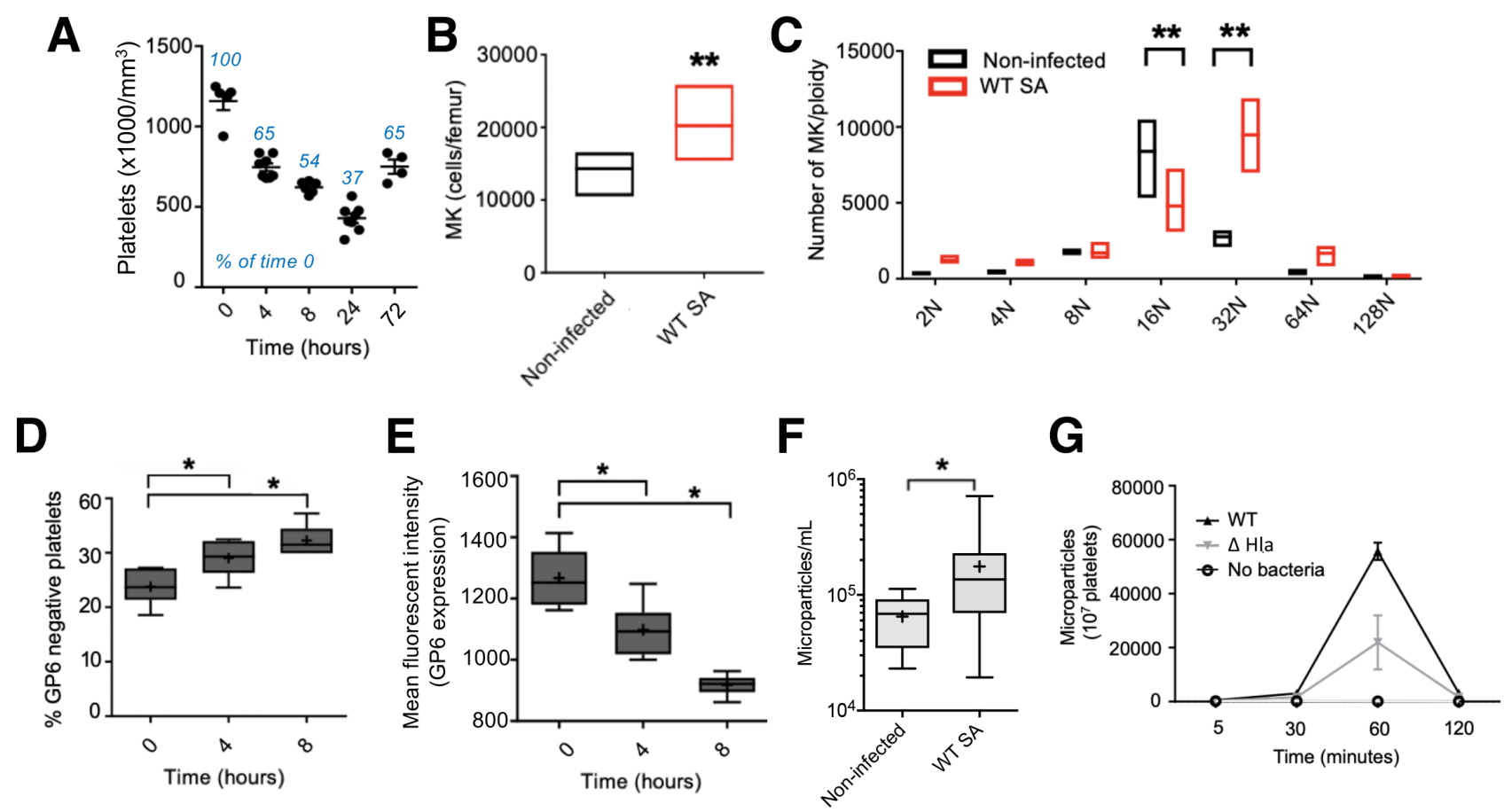

Fig. S6. Effects of SA challenge on platelets and thrombopoiesis in vivo. (A) Platelet counts after inducing systemic SA infection ( $5 \times 10^{7} \mathrm{CFU}$ SA i.v.). (B and C) Bone marrow cells were harvested after $72 \mathrm{~h}$ of SA systemic infection and analyzed for MK count (B) and ploidy distribution (C). (D and E) Circulating platelets were analyzed for \% GP6 negative platelets (D) and surface GP6 expression (E) by flow cytometry after inducing systemic SA infection $\left(5 \times 10^{7}\right.$ CFU SA i.v.). (F) Platelet microparticulation was measured in vivo. Mice were injected $1 \times 10^{8} \mathrm{SA}$ i.v. and after $4 \mathrm{~h}$, blood was collected and analyzed for platelet microparticles by flow cytometry. (G) Human platelets were infected with SA in vitro. Platelet microparticles in the samples were analyzed at each time point by flow cytometry. 


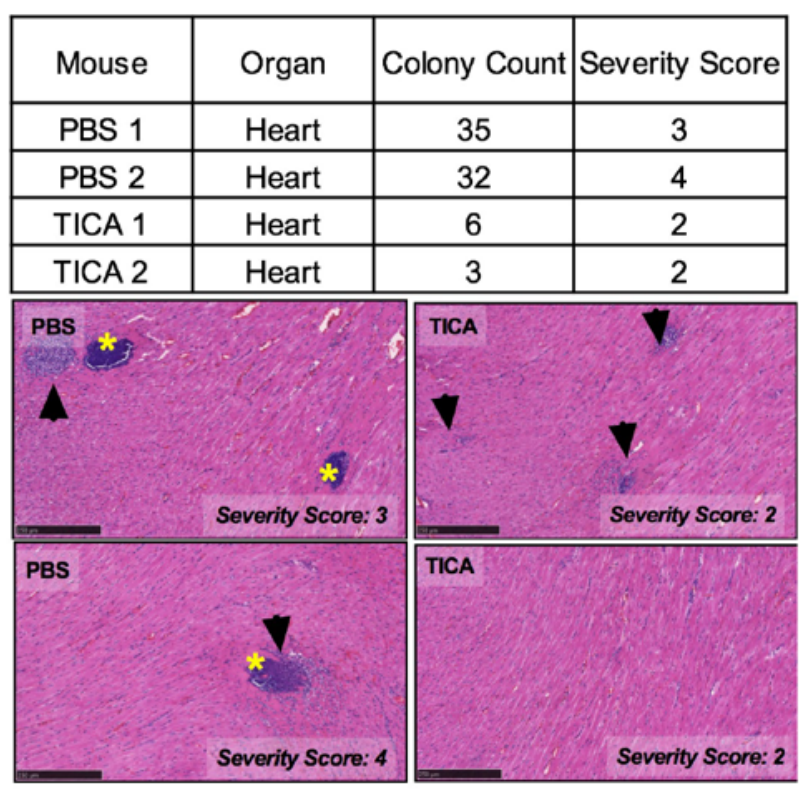

\begin{tabular}{|c|c|c|c|}
\hline Mouse & Organ & Colony Count & Severity Score \\
\hline PBS 1 & Liver & N/A & 1 \\
\hline PBS 2 & Liver & N/A & 1 \\
\hline TICA 1 & Liver & N/A & 1 \\
\hline TICA 2 & Liver & N/A & 1 \\
\hline
\end{tabular}
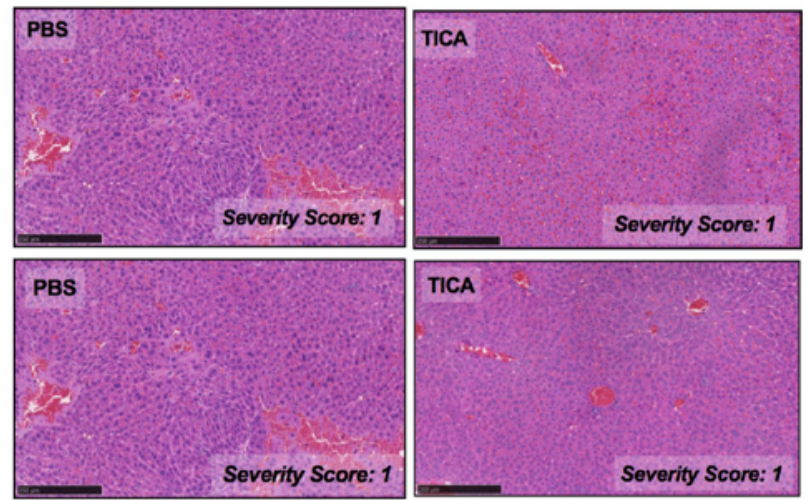

\begin{tabular}{|c|c|c|c|}
\hline Mouse & Organ & Colony Count & Severity Score \\
\hline PBS 1 & Spleen & N/A & 2 \\
\hline PBS 2 & Spleen & N/A & 3 \\
\hline TICA 1 & Spleen & N/A & 2 \\
\hline TICA 2 & Spleen & N/A & 2 \\
\hline \hline
\end{tabular}

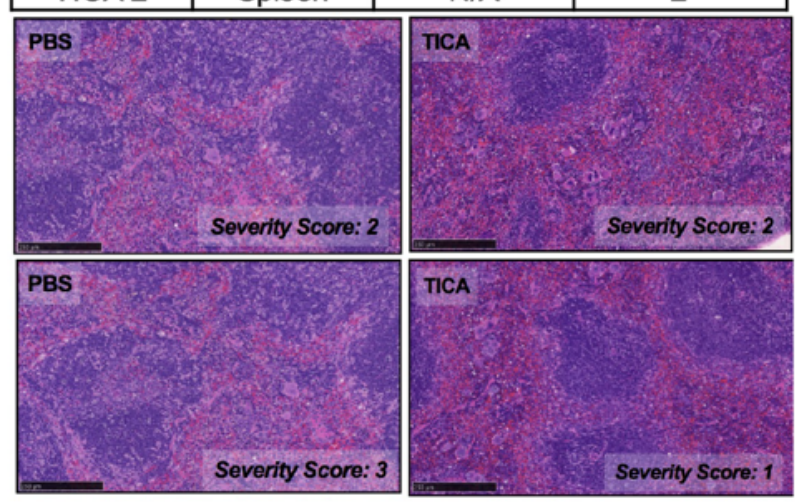

Fig. S7. Organ pathology in murine SA infection with or without ticagrelor treatment. (A) Hematoxylin and eosin stain (H\&E) of representative histological heart, liver, and spleen sections from mice pre-treated with vehicle or $4 \mathrm{mg} / \mathrm{kg}$ ticagrelor $12 \mathrm{~h}$ prior to SA infection and $\mathrm{q} 12 \mathrm{~h}$ thereafter for 72 $\mathrm{h} ;(n=2)$. Yellow stars denote dense bacterial colonies. In the Ticagrelor-treated mice, the bacterial colonies were smaller, less frequent, and often surrounded by an inflammatory infiltrate (black arrow) comprising neutrophils with fewer macrophages. Images are representative of at least two independent experiments. 

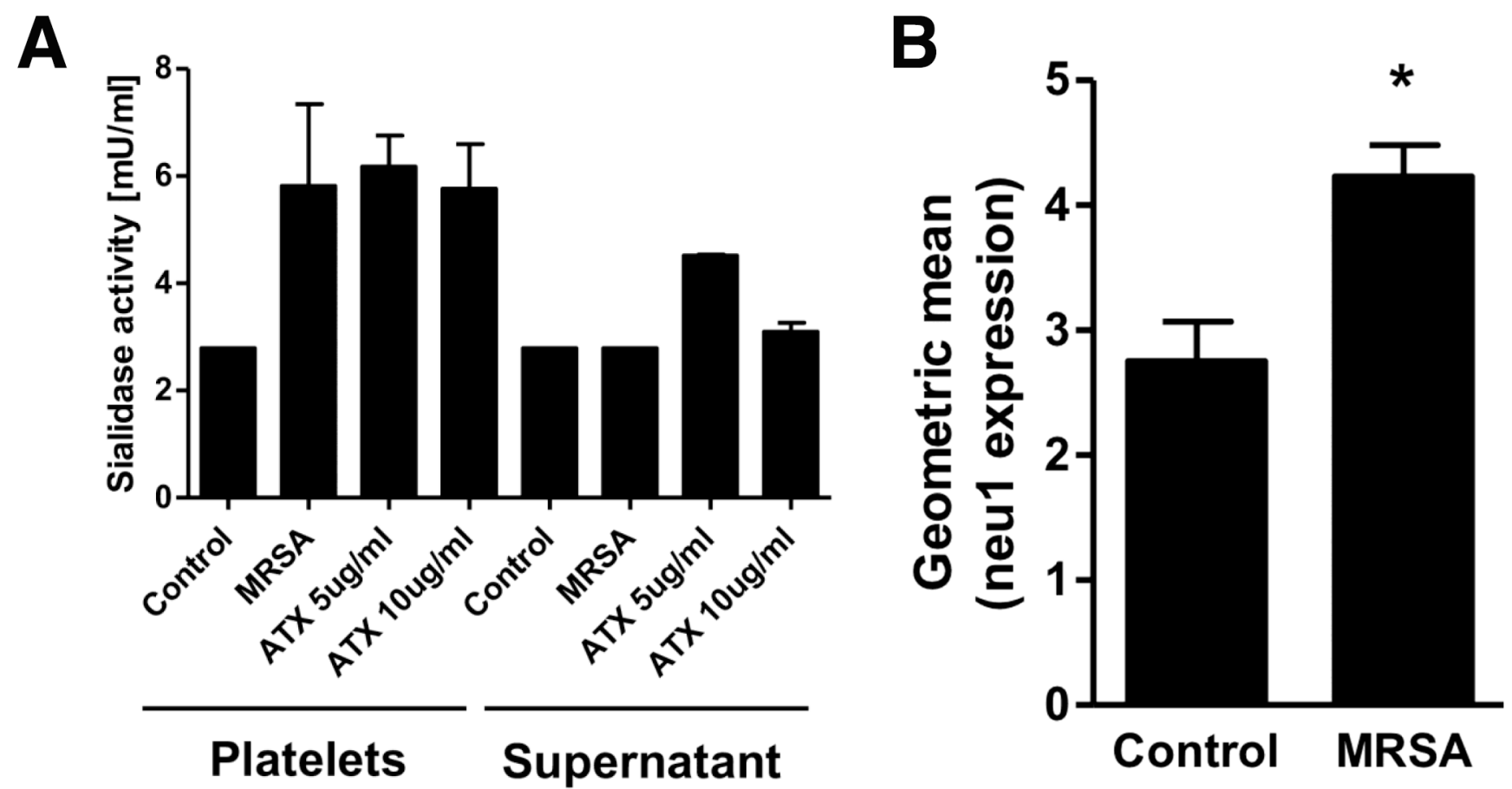

Fig. S8. Neu1 is predominantly detected on the platelet cell surface and is induced by SA exposure. (A) Human platelet-rich plasma was incubated with $2 \times 10^{7}$ CFU SA or with 5 or $10 \mu \mathrm{g} / \mathrm{ml}$ recombinant $\alpha$ toxin (ATX). Following $30 \mathrm{~min}$ incubation at $37^{\circ} \mathrm{C}$, platelets and plasma were separated by centrifugation. Both fractions were assayed for sialidase activity using the Amplyx Red Assay (Fisher Scientific) per manufacturer's instructions, using DANA as a background control. A standard curve was established using Arthrobacter ureafaciens sialidase (AUS), with a starting concentration of $500 \mathrm{mU} / \mathrm{mL}$ serially diluted down to $0.98 \mathrm{mU} / \mathrm{mL}$. (B) Human platelets were incubated with WT SA at MOI $=1$ for 30 min rotating at $37^{\circ} \mathrm{C}$, then incubated with anti-Neu1 antibody, followed by addition of AlexaFluor 488 goat anti-rabbit IgG. Samples were washed and the surface expression of Neul analyzed by flow cytometry. Data are shown as mean \pm SEM and are representative of at least three independent experiments. Statistical significance determined by unpaired Two-tailed Student's t test. $* P<0.05$ 

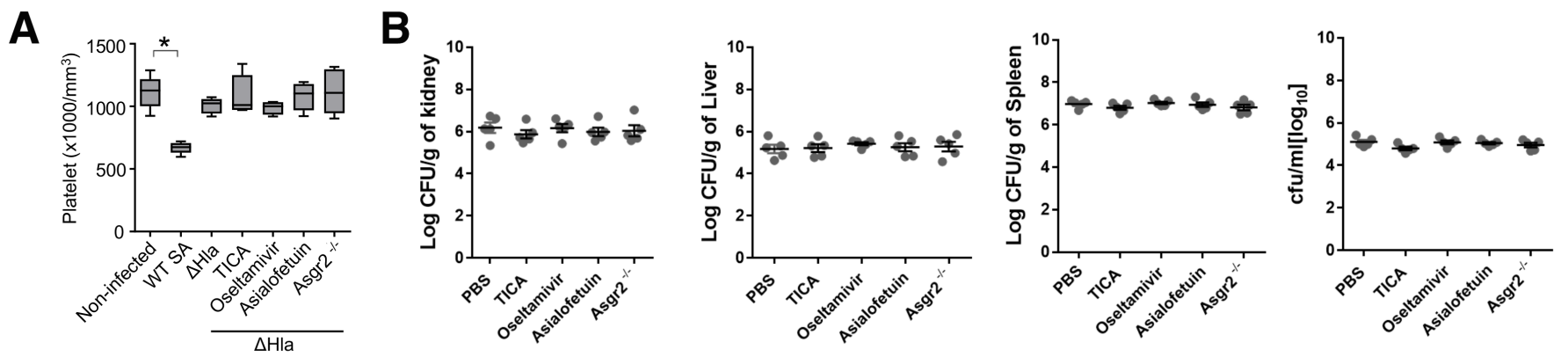

Fig. S9. SA $\alpha$-toxin deletion mutant phenocopies protective effects of TICA, oseltamivir and AMR loss/inhibition. (A) WT C57/B16 mice or Asgr2 ${ }^{-/}$mice were either challenged with $8 \times 10^{8}$ WT SA or its isogenic $\Delta$ Hla mutant. C57/BL6 mice infected with $\Delta$ Hla were subsequently treated with TICA, oseltamivir, asialofetuin, or PBS control. Blood was harvested $4 \mathrm{~h}$ post infection and (A) platelets or (B) bacterial colony forming units (CFU) enumerated. Statistical significance determined by unpaired Twotailed Student's T-test. For floating bar graphs, - denotes the median, whiskers represent min. to max, and floating box represents the 25 th to 75 th percentile. Unless otherwise stated, ${ }^{*} \mathrm{P}<0.05$. PBS, phosphate buffered saline; ns, not significant; WT, wild-type; TICA, ticagrelor. 


\section{SUPPLEMENTAL REFERENCES}

63. W. E. Rose, J. C. Eickhoff, S. K. Shukla, M. Pantrangi, S. Rooijakkers, S. E. Cosgrove, V. Nizet, G. Sakoulas, Elevated serum interleukin-10 at time of hospital admission is predictive of mortality in patients with Staphylococcus aureus bacteremia, J. Infect. Dis. 206, 1604-1611 (2012).

64. T. Bae, O. Schneewind, Allelic replacement in Staphylococcus aureus with inducible counter-selection, Plasmid 55, 58-63 (2006).

65. M. Potier, L. Mameli, M. Bélisle, L. Dallaire, S. B. Melançon, Fluorometric assay of neuraminidase with a sodium (4-methylumbelliferyl- $\alpha-d-N$-acetylneuraminate) substrate, Anal. Biochem. 94, 287-296 (1979).

66. A. J. G. Jansen, A. J. Gerard Jansen, E. C. Josefsson, V. Rumjantseva, Q. P. Liu, H. Falet, W. Bergmeier, S. M. Cifuni, R. Sackstein, U. H. von Andrian, D. D. Wagner, J. H. Hartwig, K. M. Hoffmeister, Desialylation accelerates platelet clearance after refrigeration and initiates GPIb $\alpha$ metalloproteinase-mediated cleavage in miceBlood 119, 1263-1273 (2012).

67. T. Kanaji, M.-N. Vo, S. Kanaji, A. Zarpellon, R. Shapiro, Y. Morodomi, A. Yuzuriha, K. Eto, R. Belani, M.-H. Do, X.-L. Yang, Z. M. Ruggeri, P. Schimmel, Tyrosyl-tRNA synthetase stimulates thrombopoietin-independent hematopoiesis accelerating recovery from thrombocytopenia, Proc. Natl. Acad. Sci. U. S. A. 115, E8228-E8235 (2018).

68. E. I. Park, Y. Mi, C. Unverzagt, H.-J. Gabius, J. U. Baenziger, The asialoglycoprotein receptor clears glycoconjugates terminating with sialic acida2,6GalNAc, Proc. Natl. Acad. Sci. U. S. A. 102, 17125-17129 (2005).

69. L. G. Ellies, D. Ditto, G. G. Levy, M. Wahrenbrock, D. Ginsburg, A. Varki, D. T. Le, J. D. Marth, Sialyltransferase ST3Gal-IV operates as a dominant modifier of hemostasis by concealing asialoglycoprotein receptor ligands, Proc. Natl. Acad. Sci. U. S. A. 99, 10042-10047 (2002). 